\title{
Dynamic properties of inhomogeneous states in cuprates
}

\author{
(Review Article)
}

\author{
J. Lorenzana ${ }^{1}$ and G. Seibold ${ }^{2}$ \\ ${ }^{1}$ SMC-INFM,ISC-CNR, Dipartimento di Fisica, Universitá di Roma, \\ «La Sapienza», P. Aldo Moro 2, 00185 Roma, Italy \\ ${ }^{2}$ Institut für Physik, BTU Cottbus, PBox 101344, 03013 Cottbus, Germany \\ E-mail: lorenzana.jose@gmail.com
}

Received October 26, 2005

\begin{abstract}
We review the static and dynamical properties of stripe textures with regard to their relevance in high- $T_{C}$ superconductors. Our investigations are based on the time-dependent Gutzwiller approximation which allows for the computation of random-phase approximation-like (RPA) fluctuations on top of the Gutzwiller approximation (GA). No restrictions are imposed on the charge and spin configurations which makes the method suitable for the calculation of linear excitations around symmetry-broken solutions. Within this approach we calculate the optical conductivity of stripes and compare our results with reflectivity experiments on cuprates. Furtheron we use the spin-rotational invariant extension of the GA + RPA method in order to investigate the characteristics of magnetic excitations in the stripe phase of cuprates. Our results are in very good agreement with neutron scattering and optical experiments on lanthanum cuprate superconductors.
\end{abstract}

PACS: 71.10.-w, 71.27.+a, 71.45.Gm

Keywords: textures, high- $T_{C}$ superconductivity, linear excitations.

\section{Introduction}

Early on after the discovery of high- $T_{c}$ cuprates by Bednorz and Müller [1] it was realized by both experimentalists and theoreticians that many of the unusual properties of these compounds may be understood by the assumption of an inhomogeneous distribution of charge carriers. While at the beginning this inhomogeneity was predominantly characterized as an electronically or (and) chemically driven global phase separation another point of view soon emerged which pointed out the existence of microsocopic or nanoscale inhomogeneities (for a colletion of papers on this issue see Refs. 2-4). Progress towards an understanding of the spatial structure of the inhomogeneous charge distribution was made in 1995 when Tranquada and collaborators observed a splitting of both spin and charge order peaks in $\mathrm{La}_{1.48} \mathrm{Nd}_{0.4} \mathrm{Sr}_{0.12} \mathrm{CuO}_{4}$ within elastic neutron scattering [5]. The outcome of this experiment resembled similar data in the nickelates where both in- commensurate antiferromagnetic (AF) order [6,7] and the ordering of charges $[7,8]$ has been detected by neutron scattering and electron diffraction, respectively. In Ref. 7 it was shown that the magnetic ordering in $\mathrm{La}_{2} \mathrm{NiO}_{4.125}$ displays itself as an occurrence of first and third harmonic Bragg peaks whereas the charge ordering is associated with second harmonic peaks. From this it was concluded that the doped holes arrange themselves in quasi-onedimensional structures, called stripes, which simultaneously constitute antiphase domain walls for the AF order.

Whereas the existence of static charge and spin order in the nickelates is well established by electron, neutron and x-ray diffraction [6-9] the interpretation of experimental results in the cuprate compounds [5,10-16] with respect to stripes is still controversial.

Direct evidence for static stripes in cuprates has been most clearly established in $\mathrm{La}_{2}{ }_{-} \mathrm{Sr}_{x} \mathrm{CuO}_{4}$ ( $\mathrm{LSCO}$ ) co-doped with $\mathrm{Nd}$, Eu or Ba. In this regard 
the stripe concept also helped to clarify the origin of the dip in the $T_{c}$ vs. $x$ curve at doping $x \approx 0.12$ in $\mathrm{La}_{2-x} \mathrm{Ba}_{x} \mathrm{CuO}_{4}$ [17]. However incommensurate inelastic neutron scattering signals have been found in both LSCO and $\mathrm{YBa}_{2} \mathrm{Cu}_{3} \mathrm{O}_{7-\delta}$ (YBCO) compounds with a remarkable similar phenomenology [18]. Even more static charge order has been found in YBCO without the need for additions like Nd [19]. These experiments suggest that the stripe picture applies also to LSCO and $\mathrm{YBCO}$ where stripes may have a more dynamical character.

In LSCO the inverse stripe spacing grows linearly with doping $x$ up to $x \approx 1 / 8$ and the orientation rotates from vertical (i.e., oriented along the $\mathrm{Cu}-\mathrm{O}$ bonds) by $45^{\circ}$ to diagonal for concentrations lower $x \approx 0.05[12,20,21]$. Moreover, from this linear relation it turns out that in the LSCO compounds stripes are characterized by one doped hole per two unit cells along the domain wall, that is a linear density of added holes $v \approx 0.5$ (hereafter called «half-filled stripe»).

Regarding the $\mathrm{Bi}_{2} \mathrm{Sr}_{2} \mathrm{CaCu}_{2} \mathrm{O}_{8}$ (BiSCCO) family it is a very difficult material to perform neutron scattering so few experiments exist, a notable example being Ref. 22. Interestingly this study finds a peak much broader than the momentum resolution leaving plenty of room for incommensurate effects. The existence of incommensurate scattering in BiSCCO is also supported by the experiments of Ref. 23 .

While most neutron scattering experiments focus on the structure of spin excitations, the inhomogeneous charge distribution as arising from the formation of stripes has also been detected by local probes like NQR [24-26] and NMR [27]. Due to refinements in the experimental technique Haase et al. [27] where even able to demonstrate a correlation of charge and density variations on short length scales.

All these experiments suggest that stripes are a common phenomena of all cuprates families and therefore may be related to superconductivity.

Concerning the theoretical aspects, stripes were predicted by the inhomogeneous Hartree-Fock (HF) approximation in the three-band Hubbard model [28], the one-band Hubbard [29,30] and the $t-J$ model [31]. However these pioneering studies predicted insulating stripes with a linear density of $v=1$ added holes per lattice constant along the stripe (instead of the observed $v \sim 0.5)$ which led to an early rejection of stripes [32]. It was then subsequently realized that the failure of the HF approach is due to the insufficient treatment of electronic correlations and more advanced methods have been applied to the problem. Within a density matrix renormalization group (DMRG) approach White and Scalapino [33] have found stable domain wall solutions in the physically relevant doping regime of the $t-J$ model which have the correct stripe filling. The stripe stability in the $t-J$ model has also been investigated using exact diagonalization [34,35] as well as quantum and variational Monte Carlo techniques [36,37]. Besides the HF approach a variety of methods has also been applied to Hubbard-type models in order to investigate the possibility of charge and spin order. Fleck and collaborators [38,39] have found stable metallic stripes using dynamical mean-field theory (DMFT) and shown that the spectral features of these structures are consistent with angle-resolved photoemission experiments [40]. More recently the DMRG method has also been applied to a $6 \times 7$ Hubbard model doped with four holes where a stable striped ground state was found for a large variety of $U / t$ [41]. Partially filled metallic stripes have also been stabilized within the Gutzwiller approximation [42] opening the way to studies in large systems and of fluctuation effects as will be discussed in this review.

All the above theoretical studies are based on models with only short range interactions. The tendency of strongly correlated systems towards the formation of inhomogeneous structures can also be accessed from an alternative point of view based on the concept of frustrated phase separation [43-47]. In these theories a phase separation instability is prevented by long-range Coulomb interactions. As a result the long-wavelength density fluctuations associated with phase separation are suppressed in favor of shorter-wavelength density fluctuations, giving rise either to dynamical slow density modes [43] or to incommensurate charge density waves [45-47].

The formation of stripe structures in cuprates should have profound consequences on the excitation spectra of the system in both charge and magnetic channels. If the mechanism of high temperature superconductivity is electronic the characterization of these excitation spectra is of fundamental importance in the same way as a well developed theory of phonons was fundamental for the development of Bardeen-Cooper and Schrieffer theory of superconductivity [48].

Our purpose here is therefore to give an overview over our investigations in this regard and to demonstrate that peculiar properties of a number of experiments which probe the dynamics in the charge and spin sectors can be understood within the stripe scenario based on short range models, i.e., without long-range Coulomb interaction.

Previous analogous calculations have been mostly performed within the Hartree-Fock approximation of the one-band Hubbard model supplemented with random-phase approximation (RPA) fluctuations 
[49-53]. However, in case of the HF approximation [54] vertical stripe solutions are only favored for unrealistic small values of $U / t \approx 3 . .5$ whereas a ratio of $U / t \approx 8$ is required to reproduce the low energy spectrum of the three-band model [55]. In order to enable a quantitative comparison with experiment our investigations here are based on the unrestricted Gutzwiller approximation [56,57] which provides an excellent variational Ansatz for the ground state energy of Hubbard-type models and which can also be extended to include fluctuations beyond the saddle-point solution [58-60].

The corresponding formalism is outlined in Sec. 1 where we derive the relevant RPA interaction kernels in both the charge and spin sector. For the latter, one has to go beyond the standard Gutzwiller approximation (GA) [61] since the calculation of spin excitations requires a proper consideration of transverse spin degrees of freedom. For this purpose we derive the spin-rotational Gutzwiller approximated energy functional in the appendix. In Sec. 2 we present a computation of metallic mean-field stripes [42] within the unrestricted GA applied to the three-band Hubbard model. The behavior of the magnetic incommensurability $\varepsilon \equiv 1 /(2 d)[11,12,14,15]$ ( $d$ is the distance between charged stripes in units of the lattice constant), chemical potential $[62,63]$, and transport experiments [64,65] as a function of doping is explained in a parameter free way [42]. Section 3 is devoted to an investigation of charge fluctuations based on the solutions of Sec. 2 within the time dependent GA [60]. Especially we present results for the evolution of the optical conductivity with doping [66] finding very good agreement with experiment $[67,68]$. The evaluation of magnetic excitations from stripes is the subject of Sec. 4. The complexity of the three band model as used in the previous sections, however, makes it difficult to perform such study since large system sizes have to be considered for an analysis of wave-vector dependent spin excitations. For this purpose we adopt here a much simpler Hamiltonian, namely the extended one-band Hubbard model. In the first part of Sec. 4 we derive the parameter set for this model where we require that the doping dependence of the stripe periodicity should be analogous to the results obtained within the three-band model in Sec. I. In the second part the dynamic structure factor for an underlying stripe structure is computed [69] and compared with inelastic neutron scattering data [70].

\section{Unrestricted Gutzwiller approximation and RPA fluctuations}

The Gutzwiller variational wave function together with the Gutzwiller approximation [61] is a widely used approach in order to deal with Hubbard-type models. Originally introduced in order to explore the possibility of ferromagnetism within the Hubbard model (see, e.g., Ref. 71 and references therein) its popularity resides in the fact that it captures correlation effects like the band narrowing already on the variational level. More recently the GA has been also used for realistic band structure computations [71-73]. Since in the Hubbard model one has a competition between delocalization, from the hopping of the charge carriers, and localization, from the onsite interaction $U$, the idea is to apply a projector to a given Slater determinant which reduces the number of doubly occupied sites. Within the GA one has to minimize an energy functional which is composed of a renormalized kinetic term and the interaction energy $U D$, where $D$ denotes the concentration of doubly occupied sites.

The GA can be derived using a variety of methods [61,74-77]. In particular it is recovered at the mean-field level (saddle-point) of the four-slave boson functional integral method introduced by Kotliar and Ruckenstein (KR) [77]. The latter offers the possibility of going beyond the Gutzwiller result as for example the inclusion of transversal spin degrees of freedom [78]. In addition it provides a scheme (at least in principle) to include fluctuations beyond the mean-field (MF) solution. This has made the KR method quite popular since the inclusion of fluctuations on top of the GA has been a long standing goal. Expansions around the slave-boson saddle point have been performed for homogeneous systems in Refs. 79,80 in order to calculate correlation functions in the charge and longitudinal spin channels. However, the expansion of the KR hopping factor $z^{S B}$ is a highly nontrivial task both with respect to the proper normal ordering of the bosons and also with respect to the correct continuum limit of the functional integral $[81,82]$. These problems have severely hampered practical computations of fluctuations. The time dependent Gutzwiller approximation which we present below is an alternative route to the computation of fluctuations which can be easily implemented in a variety of situations.

\subsection{Gutzwiller approximation}

In order to be specific we consider the two-dimensional Hubbard model on a square lattice, with hopping restricted to nearest $(\sim t)$ and next nearest $\left(\sim t^{\prime}\right)$ neighbors

$$
H=-t \sum_{\langle i j\rangle, \sigma} c_{i, \sigma}^{\dagger} c_{j, \sigma}-t^{\prime} \sum_{<<i j\rangle>, \sigma} c_{i, \sigma}^{\dagger} c_{j, \sigma}+U \sum_{i} n_{i, \uparrow} n_{i, \downarrow} .
$$


Here $c_{i, \sigma}^{(\dagger)}$ destroys (creates) an electron with spin $\sigma$ at site $i$, and $n_{i, \sigma}=c_{i, \sigma}^{\dagger} c_{i, \sigma} . U$ is the on-site Hubbard repulsion. The generalization of the following to higher dimensions and (or) multiband models is straightforward.

The unrestricted GA in its simplest variant can be implemented by either a variational Ansatz [83] or the Kotliar-Ruckenstein slave-boson scheme [77]. Within the variational Ansatz one constructs a Gutzwiller wave function $|\Psi\rangle$ by applying a projector to a Slater determinant $|S D\rangle$ which reduces the double occupancy. The Slater determinant has in general an inhomogeneous charge and spin distribution describing generalized spin and charge density waves [83].

The resulting energy functional $E^{G A} \equiv\langle\psi|H| \psi\rangle$ treated in the Gutzwiller approximation reads as

$$
\begin{gathered}
E^{G A}(\rho, D)=-t \sum_{<i j>, \sigma} z_{i, \sigma}^{G A} z_{j, \sigma}^{G A} \rho_{i j, \sigma}- \\
-t^{\prime} \sum_{<<i j>>, \sigma} z_{i, \sigma}^{G A} z_{j, \sigma}^{G A} \rho_{i j, \sigma}+U \sum_{i} D_{i}
\end{gathered}
$$

where $\rho_{i j, \sigma}=\left\langle c_{i, \sigma}^{\dagger} c_{j, \sigma}\right\rangle$ denotes the single-particle density matrix associated with the Slater determinant $|S D\rangle \quad$ (before Gutzwiller projection) and $D_{i}=\left\langle\Psi\left|n_{i \uparrow} n_{i \downarrow}\right| \Psi\right\rangle$ are the double occupancies. The Gutzwiller hopping factors are given by

$z_{i, \sigma}^{G A}=\frac{\sqrt{\left(1-\rho_{i i}+D_{i}\right)\left(\rho_{i i, \sigma}-D_{i}\right)}+\sqrt{D_{i}\left(\rho_{i i,-\sigma}-D_{i}\right)}}{\sqrt{\rho_{i i, \sigma}\left(1-\rho_{i i, \sigma}\right)}}$.

The energy functional Eq. (2) has to be minimized with respect to the double occupancy parameters $D$ and the density matrix $\rho$ where the latter variation has to be constrained to the subspace of Slater determinants. For technical aspects of this variational procedure we refer to Ref. 56. For unconstrained minimizations the clusters ususally have dimensions $N=N_{x} \times L$ up to $16 \times 16$ and we use periodic and antiperiodic boundary conditions in order to stabilize static and homogeneous (metallic) stripe textures. After a specific stripe texture has been found as a stable saddle-point of the GA energy functional it is also possible to investigate wave-vector dependent properties of the system. For this it is more convenient to work in $\mathbf{k}$-space and to define a Wigner-Seitz cell according to the specific stripe symmetry. In this way we can perform a constrained minimization for systems with typical dimensions $N=100 \times 100$ lattice sites.

Eq. (2) is the appropriate GA energy functional when one restricts on the longitudinal spin degrees of freedom (i.e., $S_{i}^{z}$ ). However, transverse components can be straightforwardly incorporated within the spin-rotationally invariant slave boson formulation [84]. The latter can be derived either from the KR Hamiltonian or alternatively from Gebhard's energy functional when the spin rotation is applied to the underlying Slater determinant [85]. A synthesis of both approaches is derived in appendix and yields the energy functional

$$
E^{G A}=\sum_{i, j, \sigma, \sigma_{1}, \sigma_{2}} t_{i j} z_{i, \sigma_{1}, \sigma^{z}} z_{j, \sigma, \sigma_{2}} \rho_{i j}^{\sigma_{1}, \sigma_{2}}+U \sum_{i} D_{i},
$$

where the matrix $\mathbf{z}_{i}$ reads as:

$$
\mathbf{z}_{i}=\left(\begin{array}{cc}
z_{i}^{+} \cos ^{2} \frac{\Phi}{2}+z_{i}^{-} \sin ^{2} \frac{\Phi}{2} & \frac{S_{i}^{-}}{S_{i}^{z}}\left[z_{i}^{+}-z_{i}^{-}\right] \cos \Phi \\
\frac{S_{i}^{+}}{S_{i}^{z}}\left[z_{i}^{+}-z_{i}^{-}\right] \cos \Phi & z_{i}^{+} \sin ^{2} \frac{\Phi}{2}+z_{i}^{-} \cos ^{2} \frac{\Phi}{2}
\end{array}\right),
$$

with

$$
\begin{gathered}
\tan ^{2} \Phi=\frac{S_{i}^{+} S_{i}^{-}}{\left(S_{i}^{z}\right)^{2}}, \\
z_{i}^{ \pm}=\frac{\sqrt{1-\rho_{i i}+D_{i}} \lambda_{i}^{ \pm}+\lambda_{i}^{\mp} \sqrt{D_{i}}}{\sqrt{\left(1-D_{i}-\left(\lambda_{i}^{ \pm}\right)^{2}\right)\left(\rho_{i i}-D_{i}-\left(\lambda_{i}^{\mp}\right)^{2}\right)}}, \\
\left(\lambda_{i}^{ \pm}\right)^{2}=\rho_{i i} / 2-D_{i} \pm S_{i}^{z} \sqrt{1+\tan ^{2} \Phi},
\end{gathered}
$$

and for clarity spin expectation values are denoted

$$
\begin{gathered}
\text { by } S_{i}^{+}=\rho_{i i}^{\uparrow, \downarrow}, S_{i}^{-}=\rho_{i i}^{\downarrow, \uparrow}, S_{i}^{z}=\left(\rho_{i i}^{\uparrow, \uparrow}-\rho_{i i}^{\downarrow, \downarrow}\right) / 2, \\
\text { and } \rho_{i i}=\rho_{i i}^{\uparrow, \uparrow}+\rho_{i i}^{\downarrow, \downarrow} .
\end{gathered}
$$

Regarding the stationary solutions, we will restrict to Slater determinants which are diagonal in spin space, i.e., $\rho_{i j}^{\sigma, \sigma^{\prime}(0)}=\rho_{i j}^{\sigma, \sigma(0)} \delta_{\sigma, \sigma^{\prime}}$ so that saddle-point energies can always be obtained from Eq. 2. However, in order to analyze the magnetic excitations on top of the ground state solution the energy functional Eq. 4 constitutes the appropriate starting point (cf. next subsection).

In order to estimate the quality of the GA with respect to more advanced techniques (which are however restricted to smaller clusters and specific boundaries) we have compared the energy and charge distribution with DMRG calculations [41] on a $7 \times 6$ Hubbard cluster doped with 4 holes. At the static level and $U / t=12$ the GA ground state energy is by $9-10 \%$ too large as compared to DMRG (HF is $30 \%$ too large). This lives room for fluctuation corrections to the ground state energy [60]. Remarkably the GA charge distribution is almost identical to the exact 
one. Especially both DMRG and GA result in a metallic stripe state whereas HF breaks the translational symmetry along the domain wall and produces insulating states.

\subsection{Fluctuations around the GA saddle-point}

Charge and spin excitations are obtained by computing RPA like fluctuations on top of the GA saddle-point [58-60] which fulfills standard sum rules and on small clusters yields excitation spectra in excellent agreement with exact diagonalization [59].

In the spirit of linear response theory we study an external time-dependent perturbation

$$
\begin{gathered}
F(t)=\sum_{i, j, \sigma, \sigma^{\prime}}\left[f_{i j, \sigma \sigma^{\prime}}(t) c_{i, \sigma^{\prime}}^{\dagger} j, \sigma^{\prime}+\text { h.c. },\right. \\
f_{i j, \sigma \sigma^{\prime}}(t)=f_{i j, \sigma \sigma^{\prime}}(0) \mathrm{e}^{-i \omega t},
\end{gathered}
$$

which induces small amplitude oscillations of $D$ and $\rho$ around the GA saddle point:

$$
\begin{gathered}
D=D^{(0)}+\delta D(t), \\
\rho=\rho^{(0)}+\delta \rho(t) .
\end{gathered}
$$

Correspondingly, we have to expand the energy functional Eq. (4) around the stationary solution up to second order in the density- and double-occupancy deviations. Due to the fact that we restrict to collinear saddle-point solutions, with an eventual magnetic moment oriented along the quantization axis, the fluctuations involving a spin-flip (spin) and no-spin-flip (charge) are decoupled and one obtains,

$$
E[\rho, D]=E_{0}+\operatorname{tr}\left\{h^{0} \delta \rho\right\}+\delta E^{\text {charge }}+\delta E^{\text {spin }},
$$

where the subscript 0 indicates quantities evaluated in the stationary state and we have introduced the Gutzwiller Hamiltonian [60,61]:

$$
h_{i j}^{\sigma, \sigma^{\prime}}[\rho, D]=\frac{\partial E^{G A}}{\partial \rho_{j i}^{\sigma^{\prime}, \sigma}} \delta_{\sigma, \sigma^{\prime}} .
$$

The no-spin-flip sector corresponds to excitations which do not change the total $z$ component of the spin, $\Delta S^{z}=0$. Although we identify the no-spin-flip sector with the «charge excitations» one should be aware that it includes the longitudinal magnetic excitations. The spin-flip sector with $\Delta S^{z}= \pm 1$ corresponds to the transverse magnetic excitations.

No-spin-flip sector. $\delta E^{\text {charge }}$ contains the expansion with respect to the double-occupancy parameters and the part of the density matrix, which is diagonal in the spin indices. Up to second order it is formally given by

$$
\begin{gathered}
\delta E^{\text {charge }}[\rho, D(\rho)]=E_{0}+\bar{h}_{0}^{\dagger} \delta \bar{\rho}+\frac{1}{2} \delta \bar{\rho}^{\dagger} L_{0} \delta \bar{\rho}+ \\
+\delta D S_{0} \delta \bar{\rho}+\frac{1}{2} \delta D^{t} K_{0} \delta D
\end{gathered}
$$

where the bar indicates that we are treating a matrix as a column vector and the not indicates evaluation in the stationary state, i.e., the saddle point solution. For simplicity we have skipped the notation of spin indices.

The formal complication of the present approach as compared to the standard RPA has its origin in the proper adjustment of $D$ to the time evolution of $\rho(t)$, i.e., the determination of $\delta D(t)$. This can be consistently incorporated by assuming that at each instant of time the double occupancy parameter is at the minimum of the energy functional compatible with the corresponding $\rho(t)$; i.e., the double occupancy parameters $D$ adjust antiadiabatically to the time evolution of the density matrix. This is reasonable since the double occupancy involves processes which are generally high in energy and hence fast.

From this condition of antiadiabaticity

$$
\frac{\partial E}{\partial \delta D}=0
$$

we obtain a linear relation between $\delta \rho$ and $\delta D$. Eliminating $\delta D$ from Eq. (15) finally yields an expansion of the energy as a functional of $\delta \rho$ alone $\widetilde{\delta} E[\rho] \equiv$ $\equiv \delta E[\rho, D(\rho)]$,

$$
\tilde{\delta} E[\rho]=\bar{h}_{0}^{\dagger} \delta \bar{\rho}+\frac{1}{2} \delta \bar{\rho}^{\uparrow}\left(L_{0}-S_{0}^{\dagger} K_{0}^{-1} S_{0}\right) \delta \bar{\rho} .
$$

Spin-flip sector. The spin-flip part of the expansion reads:

$$
\begin{gathered}
\delta E^{\text {spin }}=\sum_{i, j, \sigma} t_{i j} \rho_{i j}^{(0) \sigma, \sigma}\left[z_{i, \sigma, \sigma}^{0} \delta_{2} z_{j, \sigma, \sigma}+z_{j, \sigma, \sigma}^{0} \delta_{2} z_{i, \sigma, \sigma}\right]+ \\
+\sum_{i, j, \sigma} t_{i j} z_{i, \sigma, \sigma}^{0}\left[\delta_{1} z_{j, \sigma,-\sigma} \delta \rho_{i j}^{\sigma,-\sigma}+\delta_{1} z_{j,-\sigma, \sigma} \delta \rho_{j i}^{-\sigma, \sigma}\right]+ \\
+\sum_{i, j, \sigma} t_{i j} \rho_{i j}^{(0))_{\sigma, \sigma}} \delta_{1} z_{i, \sigma,-\sigma} \delta_{1} z_{j,-\sigma, \sigma},
\end{gathered}
$$

with the following abbreviations for the quadratic parts of the $z$-factor expansion

$$
\begin{gathered}
\delta_{1} z_{i, \sigma,-\sigma}=\frac{\partial z_{i, \sigma,-\sigma}}{\partial \rho_{i i}^{-\sigma, \sigma}} \delta \rho_{i i}^{-\sigma, \sigma}, \\
\delta_{2} z_{i, \sigma, \sigma}=\frac{\partial^{2} z_{i, \sigma, \sigma}}{\partial \rho_{i i}^{\sigma,-\sigma_{2}} \partial \rho_{i i}^{-\sigma, \sigma}} \delta \rho_{i i}^{\sigma,-\sigma} \delta \rho_{i i}^{-\sigma, \sigma} .
\end{gathered}
$$


It is interesting to observe that, in contrast to the charge excitations, the evaluation of the magnetic excitations can be performed without any adjustment of $\delta D$ to $\delta \rho$, i.e., without any assumption on the time evolution of $D$. Only in the case of non-colinear saddle points one would have a coupling between spin and charge fluctuations and, therefore, the necessity to invoke the antiadiabaticity condition to eliminate the $\delta D$ deviations.

In a paramagnet the ground state is a singlet and the lowest magnetic excitations corresponds to a triplet with $\Delta S^{z}=0, \pm 1$. One can show [59] that the above expansions in the spin-flip and no-spin-flip sectors correctly reproduce the degeneracy of longitudinal (no-spin-flip, $\Delta S^{z}=0$ ) and transverse (spin-flip, $\left.\Delta S^{z}= \pm 1\right)$ magnetic excitations. In Ref. 59 we have interpreted this as an additional support for the antiadiabatic condition. More recently we have realized that also the longitudinal excitations from the no-spin-flip sector decouple from the doubble occupancy fluctuations. As a consequence this degeneracy can not be invoked as an extra support for the antiadiabatic condition. This leaves some freedom for how to treat the time dependence of the doubble occupancy fluctuations and one can envisage a more elaborate formalism which may take into account retardation effects without violating standard sum rules which are obeyed within the present scheme.

Eqs. (17) and (18) can be regarded as the expansion of an effective interacting energy functional in which the interaction potential between particles is density dependent. This kind of functional often appears in the context of nuclear physics and a well developped machinery exist to compute the RPA fluctuations induced by the interaction. Details of the corresponding formalism can be found in Refs. 86,87 . The advantage of this method with respect to other methods (eg. diagrammatic) is that the present derivation is solely based on the knowledge of an energy func-

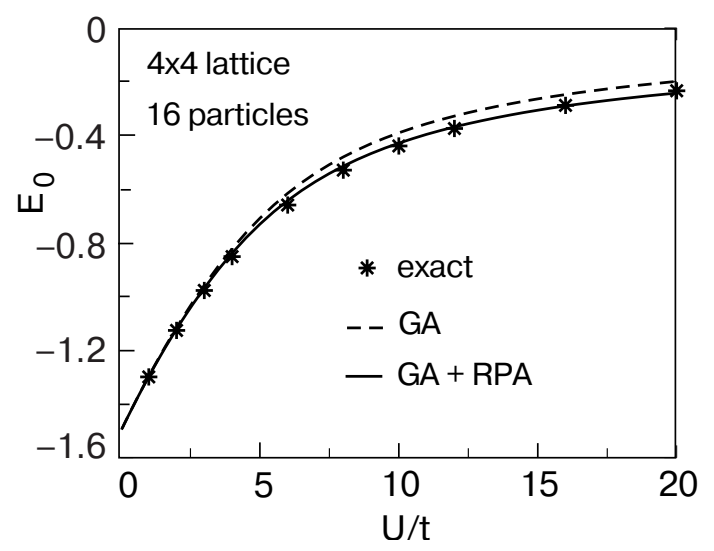

Fig. 1. Comparison of the exact ground state energy [89] with the GA and GA+RPA method for the half-filled Hubbard model on a $4 \times 4$ system. tional of a SD density matrix which is precisely what the GA provides.

In order to demonstrate the quality of the method and its improvement over the traditional HF + RPA approach we present some selected results for the half-filled Hubbard model in the antiferromagnetic Néel state [88]. The double occupancy at the RPA level is given by: $D_{R P A}==\int d \omega \sum_{\lambda}\left\langle 0\left|n_{i \uparrow}\right| \lambda\right\rangle\left\langle\lambda\left|n_{i \downarrow}\right| 0\right\rangle \delta\left(\omega-E^{(\lambda)}\right)$ where the integrand is the Lehmann representation of an appropriately defined density-density correlation function. The matrix elements $\left\langle 0\left|n_{\uparrow}\right| \lambda\right\rangle$ for $\lambda>0$ can be computed in terms of the eigenvectors $V^{(\lambda)}[86,87]$.

From the interaction energy $U D_{R P A}$ we compute the correction to the ground state energy using the coupling constant integration trick [88]. In order to compare with exact results [89] we consider a $4 \times 4$ cluster and find very good agreement up to very large values of $U / t$ as shown in Fig. 1. The improvement with dimensionality is expected as in any MF + RPA computation.

Fig. 2 shows the magnetic excitation energies for the same cluster as a function of $U / t$ evaluated within the GA + RPA, the HF + RPA and the exact diagonalization. Note that the $4 \times 4$ system has a further accidental symmetry, which causes degeneracy between the $q=(\pi / 2, \pi / 2)$ and $q=(\pi, 0)$ excitations. Furthermore, the SDW ground state of the GA and HF solution leads to the doubling of the Brillouin zone so that, besides the antiferromagnetic wave vector $Q$, only $q=(\pi / 2,0)$ and $q=(\pi, 0)$ correspond to independent excitations. On the other hand, on the $4 \times 4$ lattice, we have that the exact energies at $q=(\pi / 2,0)$ and $q=(\pi / 2, \pi)$ are slightly different.

The small- $U$ behavior of the lowest excitation energy in Fig. 2 can be well understood from the SDW

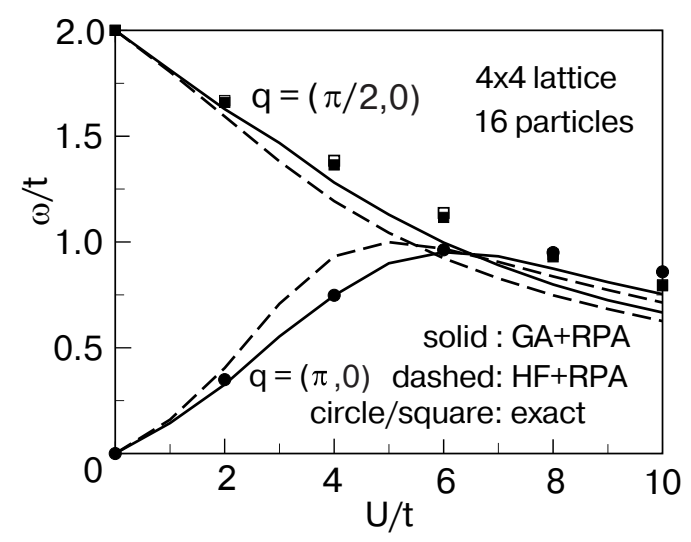

Fig. 2. Magnetic excitations at $q=(\pi / 2,0)$ and $q=(\pi, 0)$ as a function of $U / t$ for a half-filled $4 \times 4$ cluster: GA + RPA (solid line), HF + RPA (dashed line), and exact diagonalization (full circles and full squares). The exact diagonalization results for the excitation at $q=(\pi / 2, \pi)$ are also reported (empty squares). 
picture. Within this approximation, the band structure in the reduced Brillouin zone is given by $E_{q}= \pm \sqrt{\varepsilon_{q}^{2}+\Delta^{2}}$, with $\varepsilon_{q}=-2 t\left[\cos \left(q_{x}\right)+\cos \left(q_{y}\right)\right]$ and $\Delta$ denotes the SDW gap. Since we study a half-filled system, all states with $E_{q}<0$ are occupied. Consider first the $q=(\pi, 0)$ excitation which can be attributed to a spin-flip transition from $q_{1}=$ $=(-\pi / 2, \pm \pi / 2)$ to $q_{2}=(\pi / 2, \pm \pi / 2)$ so that the excitation energy is given by $\omega=E_{q_{1}}-E_{q_{2}}=2 \Delta$. The SDW gap in the HF approximation is related to the on-site magnetization $\Delta^{H F}=2 U\left|S_{z}\right|$, whereas within the KR formulation of the GA it is determined by the difference in the local spin-dependent Lagrange multipliers $\Delta^{G A}=\lambda_{\uparrow}-\lambda_{\downarrow}$. Since in the limit $U \rightarrow 0$ the GA reduces to the HF approximation, both excitation energies coincide in this regime and also agree with the exact result. On the other hand, for $U \gtrsim 1$, where RPA corrections become important, it can be seen from Fig. 2 that the GA + RPA is in much better agreement with exact diagonalization than the corresponding HF + RPA result. As a consequence, the $\mathrm{GA}+\mathrm{RPA}$ gives a quite accurate description of the crossover (at $U / t \approx 6$ ) from the SDW regime, where a gap proportional to $U$ opens along the Fermi surface, to the Heisenberg regime, where there are low-energy magnetic excitations with energy scale $t^{2} / U$.

For the higher energy triplet excitation at $q=(\pi / 2,0)$, the GA + RPA yields energies which are slightly lower than the exact result. However, whereas the discrepancy for the GA $+\mathrm{RPA}$ at $U / t=6$ is around $10 \%$, the HF + RPA deviates by almost $20 \%$ from the exact diagonalization result.

\section{Stripes in the three-band Hubbard model}

Whichever is the specific property of stripes to be analyzed a one- or three-band description may be the more suitable one. For example, direct interband excitations are not important for the description of the low energy magnetic fluctuations so that for the investigation of the latter (cf. Sec. 4) a one-band description is the more appropriate one. The one-band model is also convenient for analyzing the generic properties of stripes within the GA and to elucidate differences to the standard HF approximation [91]. On the other hand the three-band hamiltonian provides a more solid basis for computing ground state energies of different inhomogeneous textures the stability of which has to be compared in order to obtain reliable saddle-points for a random-phase computation. Also the analysis of experiments which probe local density distributions on $\mathrm{Cu}$ and $\mathrm{O}$ obviously has to be performed within the three-band approach. We do not address here the im- portant question under which circumstances the three-band model can be mapped onto the one-band hamiltonian. Rather we take the more pragmatic point of view that both models have their own eligibilty depending on the specific property of high- $T_{C}$ cuprates to be analyzed.

In the following we start therefore by examining the stabilty of stripe textures within the three-band Hubbard model. It turns out that in the underdoped regime this approach yields so-called bond-centered (BC) textures as the most stable stripe configuration whereas at higher doping site-centered (SC) structures compete. Later on in Sec. 3.2 we will then compute and analyze the optical conductivity of the three-band model based on these stripe saddle-points where the doping dependent degeneracy of stripe structures will have important consequences on the low energy charge response. In Sec. 4 the stability of stripes is also analyzed in the one-band model. Special attention will be paid to the derivation of a consistent parameter set which allows us to calculate the magnetic excitations of stripes and to compare with neutron scattering experiments.

\subsection{Phase diagram and stability of stripes}

Our starting hamiltonian is the three-band Hubbard model

$$
\begin{gathered}
H=-t_{p d} \sum_{<i j>}\left(d_{i \sigma}^{\dagger} p_{j \sigma}+p_{j \sigma}^{\dagger} d_{i \sigma}\right)+\varepsilon_{d} \sum_{i} n_{i}^{d}- \\
-t_{p p} \sum_{<j j^{\prime}>}\left(p_{j \sigma}^{\dagger} p_{j^{\prime} \sigma}+p_{j \sigma^{\prime}{ }^{\dagger}}^{\dagger} p_{j \sigma}\right)+\varepsilon_{p} \sum_{j} n_{j}^{p}+ \\
+U_{d} \sum_{i} n_{i \uparrow}^{d} n_{i \downarrow}^{d}+U_{p} \sum_{j} n_{j \uparrow}^{p} n_{j \downarrow}^{p}+U_{d p} \sum_{<i j>} n_{i}^{d} n_{j}^{p}
\end{gathered}
$$

where the fermion operators act on the vacuum configuration $\mathrm{Cu}\left[d^{10}\right] \mathrm{O}\left[p^{6}\right]$ in the hole representation. The brackets $\langle\ldots\rangle$ denote summation over nearest neighbors and the $\mathrm{Cu}-\mathrm{O}$ and $\mathrm{O}-\mathrm{O}$ hopping matrix elements include the phase factors due to the $d$ and $p$ symmetry of the $\mathrm{Cu}$ and $\mathrm{O}$ orbitals. The local orbital levels are denoted by $\varepsilon_{d}$ and $\varepsilon_{p}$ and the charge-transfer energy is $\Delta=\varepsilon_{d}-\varepsilon_{p} . U_{d}, U_{p}$ and $U_{d p}$ are the Hubbard interactions on and between nearest $\mathrm{Cu}$ and $\mathrm{O}$ sites respectively. We consider the following parameter set as deduced from local density calculations of McMahan et al. [92]: $t_{p d}=1.5 \mathrm{eV}, \quad t_{p p}=0.6 \mathrm{eV}, \quad \Delta=3.3 \mathrm{eV}$, $U_{d}=9.4 \mathrm{eV}, U_{p}=4.7 \mathrm{eV}, U_{p d}=0.8 \mathrm{eV}$. Other standard sets obtained from $a b$ initio calculations (see e.g. Ref. 55,93) do not change the results.

Since $U_{d}$ is the largest energy scale in the hamiltonian we treat the $\mathrm{Cu}$ on-site correlation within 
Site-centered

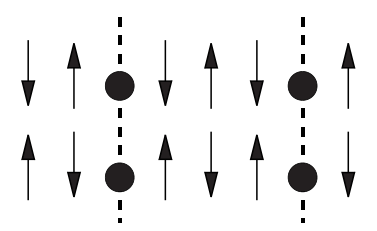

Bond-centered

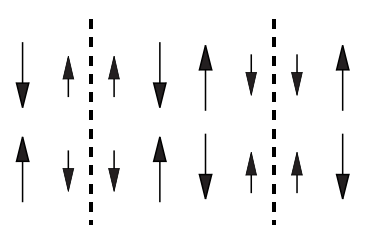

Fig. 3. Sketch of $d=4$ bond- and site centered stripe structures. Only $\mathrm{Cu}$ sites are shown. The dashed lines indicate the antiphase domain boundary.

the Gutzwiller approximation and decouple the residual interactions via Hartree-Fock. The resulting energy functional is then minimized according to the procedure described above where we ususally start with a HF stripe Slater determinant. Following the standard terminology from one band models $[33,39]$ the stripe structures can be classified in bond- and site centered (BC, SC) which differ by the location of the antiphase domain wall separating the $\mathrm{AF}$ regions (cf. Fig. 3). In the three band model SC solutions are centered on $\mathrm{Cu}$ and $\mathrm{BC}$ solutions are centered on $\mathrm{O}$. All vertical stripe solutions investigated are metallic (i.e., homogeneous along the stripe) except for integer $v$ and we have suppressed $1-\mathrm{D}$ instabilities by choosing appropriate boundary conditions (i.e., periodic or antiperiodic in direction of the stripes).

Figure 4 depicts the cross section of a typical charge and spin distribution of so-called bond- and site centered (BC, SC) vertical stripes. BC stripes are built up from two $\mathrm{Cu}-\mathrm{O}$ legs and the antiphase boundary corresponds to the $O_{x}$ sites between the two legs. On the other hand the antiphase boundary for SC textures is on a single $\mathrm{Cu}-\mathrm{O}$ leg so that by symmetry the spin density vanishes on these sites. For both structures added

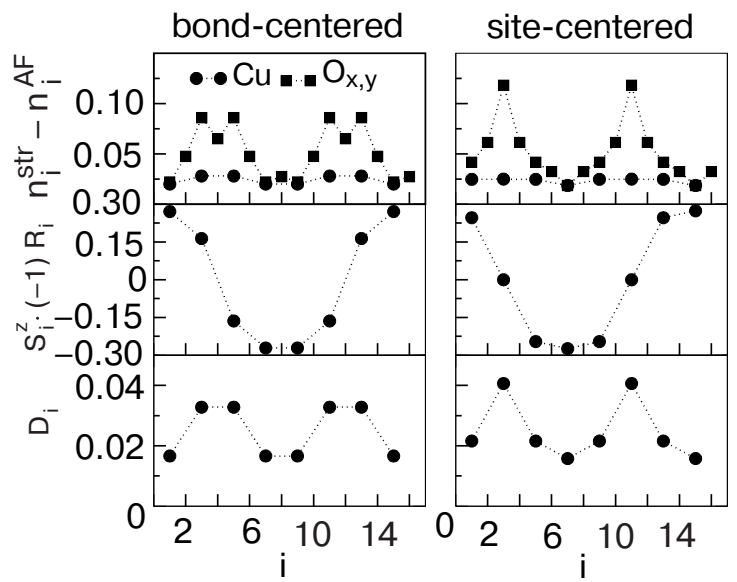

Fig. 4. Cross section of charge- and spin densities for $d=$ $=4 \mathrm{BC}$ and $\mathrm{SC}$ stripes in the three-band model. Charge (hole) densities are shown as differences between the stripe solution and the homogeneous antiferromagnet. The lowest panels report the double occupancies on $\mathrm{Cu}$. charge is mainly accumulated on the oxygen sites near (and on) the antiphase boundary between the AF regions. The hole density on the $\mathrm{Cu}$ sites does not vary significantly but nevertheless the symmetry of the stripe state reflects in the $\mathrm{Cu}$ double occupancy (lowest panels in Fig. 4). It is exactly this decoupling of charge- and spin densities from the variational double occupancy parameter which leads to an improvement of the GA over the tradional HF approximation [91].

In Fig. 5 we show the band structure (corresponding to BC stripes separated by $d=4$ unit cells) for the electrons in an extended zone scheme. Two bands appear well inside the charge transfer gap. The upper one at $\sim 0.9 \mathrm{eV}$ is more $\mathrm{Cu}$ like and quite flat (even in the stripe direction) whereas the lower one has substantial dispersion, is mainly $\mathrm{O}$ like, and crosses the Fermi level. Counting both ingap bands the $v=1 / 2$ system corresponds to $3 / 4$ hole $(1 / 4$ electron $)$ band filling. The gap between the bands is related to the magnetic character of the stripe and is absent in SC solutions with a non-magnetic core. The electronic structure close to the chemical potential is well represented by a half-filled cosine like band, hereafter referred to as the active band.

For large stripe separation the dispersion of the active band perpendicular to the stripe is quite flat indicating quasi $1 \mathrm{D}$ behavior. Small oscillations appear in this direction for $d \leq 5$ which can be identified as a crossover interstripe distance from 1D to 2D behavior and corresponds to a crossover doping $x=0.1$ for $v=0.5$.

The band structure in the stripe direction (right panel) is quite similar in all BC stripes solutions regardless of $d$ which instead determines the periodicity in the perpendicular direction (left panel). Similar electronic

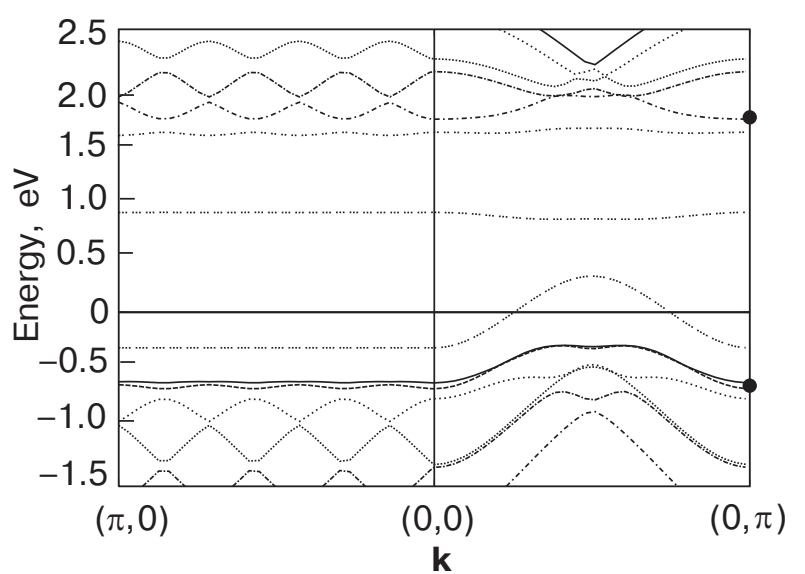

Fig. 5. Electron mean-field bands for the BC stripe of Fig. 4 measured from the chemical potential. Left (right) panel is in the direction perpendicular (parallel) to the stripe. We also plot the insulating charge transfer gap at momentum $\mathbf{k}=(0, \pi)$ measured from the same reference energy (dots). 
structures of a striped state have been obtained by DMRG $[38,39]$. Note that the Fermi surface crossing at vertical momentum $k_{y} \approx \pi / 4$ and the associated flat portion of the active band in the direction perpendicular to the stripe correlates well with the flat bands observed in many cuprates around $\mathbf{k} \approx(\pi, 0)$ [94].

To evaluate the stability we compute the energy per added hole for $N_{h}$ holes added to the system with $N$ $\mathrm{CuO}_{2}$ units,

$$
e_{h} \equiv\left[E\left(N+N_{h}\right)-E(N)\right] / N_{h} .
$$

Here $E\left(N+N_{h}\right)[E(N)]$ is the total energy of the doped [undoped AF] solution. In Fig. 6, $a$ we show $e_{h}$ as a function of the filling fraction $v \equiv N_{h} /\left(N_{s} L\right)$ for $\mathrm{BC}$ vertical stripes. Each curve corresponds to a fixed $d$. The curves have a sharp minimum at $v=0.5$. This is in contrast to early one-band [30,31,94] and three-band mean-field computations [28] for which $v=1$ is the most favorable hole filling. The discrep-
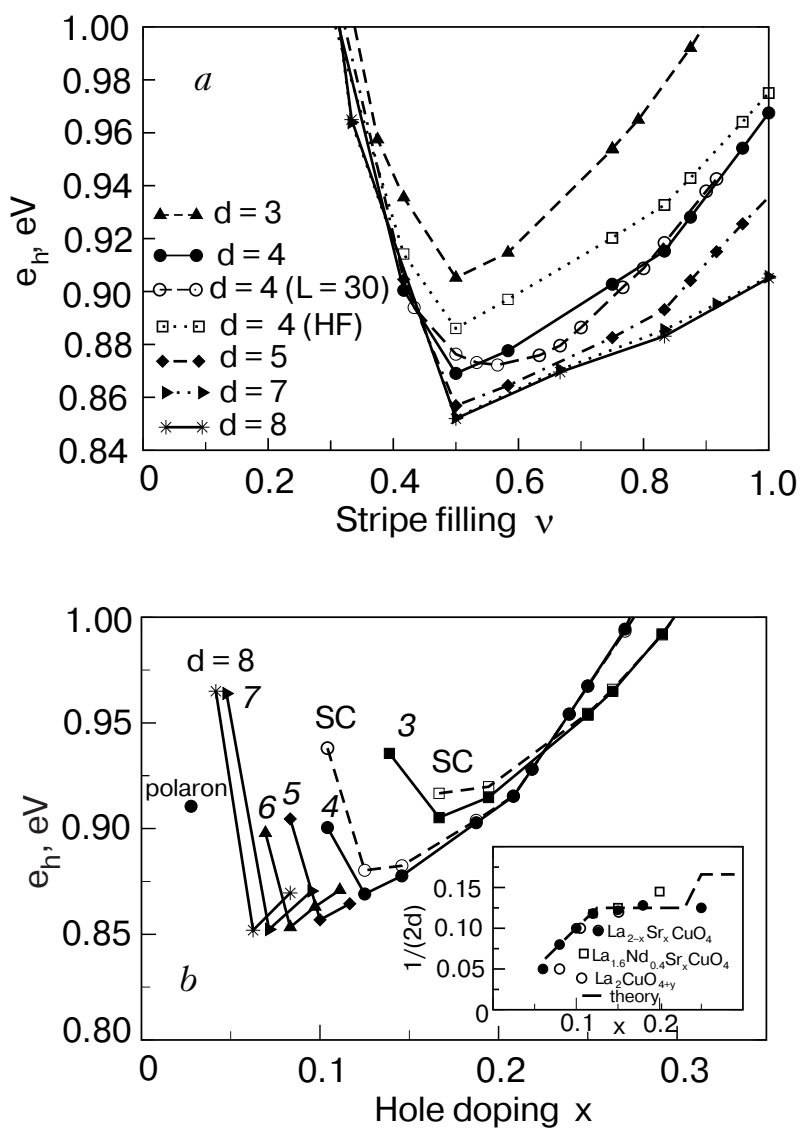

Fig. 6. $e_{h}$ as a function of $v(a)$ and $x(b)$ for vertical BC stripes. Sizes are $4 d \times 12$ for $d \leq 6$ and $2 d \times 12$ for $d>6$. In (a) we also show the result for $4 d \times 30$ (open circles). In (b) we also show the result for site-centered stripes (labeled SC, open symbols) for $d=3,4$ and one self-trapped hole or «electronic polaron» at $x=1 / 36$. The inset in (b) reports the incommensurability as obtained from the present calculation (line) compared with experimental data from Ref. 12. ancy can be traced back to the missing of a direct oxygen-oxygen hopping $\left(t_{p p}=0\right)$ in the parameter set of Ref. 28. We will come back to the importance of this parameter in the framework of the one-band model in Sec. 4 where it can be introduced as a next-nearest neighor hopping $t^{\prime}$.

Vertical stripes should be compared with other possible ground states. We find that within the present GA approach they are lower in energy than polaron solutions [96] (see Fig. 6,b) and diagonal stripes solutions (not shown). Very low nonsuperconducting dopings $(x<0.05)$ were not explored in detail since we believe that a careful consideration of other effects is required in this case. Especially long-range order perpendicular to the planes favors loop configurations of stripes [33] and long-range Coulomb effects [44,47] are expected to become important. The increased orthorombicity at low doping also indicates the importance of the lattice in determining the structure of inhomgeneous textures.

If one uses the $\mathrm{HF}$ approximation instead of the GA the minimum also occurs at $v<1$ for $\mathrm{BC}$ vertical stripes provided one uses the present parameter set. The insulating stripes found in Ref. 28 with the wrong filling $v=1$ are due to a parameter set with $t_{p p}=0$. However a polaron lattice is the ground state in HF and diagonal stripes are lower in energy than vertical ones. Thus the overall success of the present mean-field computation with respect to the earlier ones [28] is due to both a more accurate mean-field approximation GA instead of HF) and a more accurate parameter set.

Further stabilization of the half-filled stripes can occur if due to many-body effects a gap or pseudogap tends to open at the commensurate filling $v=1 / 2$ [95]. Although we do not need this effect to explain $v \approx 1 / 2$ it is quite possible that this produces a fine tuning for $T<T^{*}$. In this regard it is interesting to remark that the $\ll \mathrm{V} »$ shape form of the curves in Fig. 6, $a$ is due to the gap produced by the discretization of the levels in a finite system. For larger systems (see $d=4$ curve in Fig. 6,a) the cusp becomes rounded, however the minimum is still close to $v=1 / 2$. One should be aware that the curves with the cusp are for already quite long stripes $(L=12)$ and it is not clear whether stripes in real materials will be much longer. Thus finite size data could turn out to be more realistic than infinite size one. Within experimental and theoretical finite size errors our optimal filling fractions are in good agreement with the ones found at low superconducting dopings $[5,10-12,14,15]$.

For $d>4$ the curves coincide close to $v=0.5$ in Fig. $6, a$ whereas for $d=3,4$ the curves are shifted up. This feature is due to the width of the domain wall of $4 \sim 5$ 
lattice sites (see Fig. 4) which forces stripes to overlap when $d<5$ leading to an increase of $e_{h}$.

In Fig. 6,b we show $e_{h}$ for various values of the stripe separation $d$ and as a function of doping $x$ assuming each dopant introduces one hole. Since $x=\mathrm{v} / d$ curves with larger $d$ appear at lower concentration. The locus of the minimum of $e_{h}$ as a function of doping in Fig. 6,b is expected to form a continuous curve in the thermodynamic limit by combining different $d$ solutions in new solutions with larger periodicity. Up to $x \approx 1 / 8$ the stripe filling is fixed at $v \approx 0.5$ and consequently the density of stripes increases with doping. This explains the behavior of the incommensurability $\varepsilon=x /(2 v) \approx x$ as seen in neutron scattering experiments in this doping range (inset of Fig. 6) $[5,11,12,14,15]$. For $x>1 / 8$ the right branch of the $d=4$ solution is more stable than the $v \approx 0.5$ and $d=3$ solution due to the stripe overlap effect. Therefore the incommensurability remains locked at $\varepsilon \approx 1 / 8$ in good agreement with the change of behavior in $\varepsilon$ observed around $x \approx 1 / 8[11,12,14,15]$.

As doping increases further, BC stripes become degenerate with $\mathrm{SC}$ ones at $x \approx 0.21$. This degeneracy between $\mathrm{O}$-centered and $\mathrm{Cu}$-centered stripes is also found in many other approaches [33,39] and suggests that as doping increases lateral fluctuations of the stripe will become soft. This is interesting given the possibility that the lateral fluctuations may mediate pairing between holes. In Sec. 3.2 it will be shown that this feature has important implications for collective excitations which e.g. reflects as a softening of certain modes in the optical conductivity.

For doping $x>0.225$ we find the $d=3$ stripe ( $\varepsilon=1 / 6 \approx 0.17$ ) to become the lowest energy solution with an initial filling fraction $v=0.675$. Experimentally the situation is not clear. $\varepsilon=1 / 6$ has been reported for $\mathrm{YBa}_{2} \mathrm{Cu}_{3} \mathrm{O}_{6+\delta}$ [15] (YBCO) but not for LSCO where $\varepsilon$ remains in the $\varepsilon=1 / 8$ line up to $x=0.25$ (inset of Fig. 6). In $\mathrm{La}_{2-x-y} \mathrm{Nd}_{y} \mathrm{Sr}_{x} \mathrm{CuO}_{4}$ (LNSCO), where stripes are pinned by the low-temperature tetragonal lattice distortion, the incommensurability is substantially increased beyond $\varepsilon=1 / 8$ at doping $x=0.2$ but without reaching $\varepsilon=1 / 6[11]$.

It is possible that lateral stripe fluctuations become so strong at this doping range that an effectively isotropic state is reached [97]. Another possibility is that the $d=3$ phase is skipped due to phase separation among the $d=4$ phase and the overdoped Fermi liquid which for our parameters becomes the lowest energy solution close to $x=0.4$ (see Ref. 98 for a related scenario). Further theoretical and experimental work should be done to clarify this point. Especially being the system charged a careful analysis of phase separation is needed [46,47].
It has been emphasized [63] that there is a close connection between the doping dependent incommensurability as discussed above and the chemical potential in cuprates. In fact the chemical potential for the electrons can be related to $e_{h}$ via: $\mu=$ $=-\left(e_{h}+x \partial e_{h} / \partial x\right)$. From Fig. 6,b one can then deduce that $\mu$ is approximately constant for $x \lesssim 0.1$ and decreases for $x \gtrsim 0.1$ in qualitative agreement with the observed behavior [62,63]. The rate of change of $\mu$ with doping, being a high derivative of the energy, is very sensitive to finite size effects and, moreover, few experimental points are available in this doping range in order to allow for a precise comparison. A rough estimate indicates that the theoretical rate of change of $\mu$ with doping for $x>1 / 8$ is approximately a factor of 2 larger than the experimental one $[62,63]$. This may be attributed to an underestimation of the mass renormalization in mean-field. Another possibility which goes in the right direction is phase separation among the $d=4$ stripe solution and the paramagnetic overdoped Fermi liquid as mention above.

We finally turn to the discussion of our calculation in light of measurements of the Hall coefficient $R_{H}$ in LNSCO [64] and YBCO [65]. In the former compound $R_{H}$ displays an abrupt decrease below the charge-stripe ordering temperature $T_{0}$ and for concentrations $x \leq 1 / 8$. It has convincingly been argued that quasi $1 \mathrm{D}$ transport is not enough to explain this anomaly and instead reflects a remarkable cancellation due to particle-hole symmetry in the stripe state $[65,99]$. A partial suppression of $R_{H}$ (and simultaneously the thermopower $S$ ) below some temperature $T_{\max }$ has also been observed in YBCO up to oxygen contents corresponding to doping $x \sim 1 / 8$ [65].

For distant stripes we indeed observe that the chemical potential crosses an approximately particle-hole symmetric band (see Fig. 5) providing a natural explanation to the simultaneous suppression of $R_{H}$ and $S$. Interstripe hopping becomes significant for $x>0.1$. However it does not break particle-hole symmetry. As discussed above the chemical potential shifts from the center of the band for $x>1 / 8$, thus breaking particle-hole symmetry and consequently both $R_{H}$ and $S$ start to grow in modulus explaining the crossover behavior observed. Unfortunately the magnitude and even the sign of the transport coefficients cannot be predicted by a knowledge of the band structure alone. For example $R_{H}$ for our Fermi surface (which turns out to be open) is determined by the unknown anisotropy of the scattering path length [100].

\subsection{Optical conductivity}

The doping-dependent evolution from insulating behavior to a strange metal in the superconducting 
cuprates emerges dramatically in the normal state optical conductivity. Slightly doped cuprates show a small or no Drude peak and doping-induced transfer of spectral weight from the charge-transfer $(\sim 2 \mathrm{eV})$ to a mid-IR (MIR) band at $\sim 0.5 \mathrm{eV}[67,68]$. Upon further doping the system progressively metallizes as is evident from the prominent Drude-like peak that develops at zero energy. A remarkable effect of doping is that the MIR band strongly softens and merges with the Drude peak, resulting in a feature that cannot be fitted by a conventional Drude model. A variety of alternative theories $[101,102]$ have been proposed in order to describe this feature. Clearly the identification of this low-energy MIR (LEMIR) band is of paramount importance to understand the physics of these materials. The low-doping behavior has been explained in terms of the random-phase-approximation (RPA) electronic excitations of single-hole Hartree-Fock states in $\mathrm{CuO}_{2}$ layers [96], but the moderate doping behavior (the softening of the LEMIR band) could not be explained due to difficulties with the HF ground state.

The softening of the LEMIR band in $\mathrm{La}_{2-x} \mathrm{Sr}_{x} \mathrm{CuO}_{4}$ (LSCO) is accompanied by the appearance of another (much less discussed) band at $1.3 \mathrm{eV}$ $[67,68,103]$. This high energy MIR (HEMIR) band is well pronounced in optical absorption through LSCO thin films [68], and electron energy loss spectroscopy [103] where it develops as a function of doping. Moreover LEMIR and HEMIR are also detected in photodoped experiments on LSCO [104]. The HEMIR has not been clearly resolved by reflectivity in $\mathrm{YBa}_{2} \mathrm{Cu}_{3} \mathrm{O}_{6+\delta}(\mathrm{YBCO})$, but a strong broad feature at the right energy appears in photodoped transmission experiments [105]. As far as we know no micro-
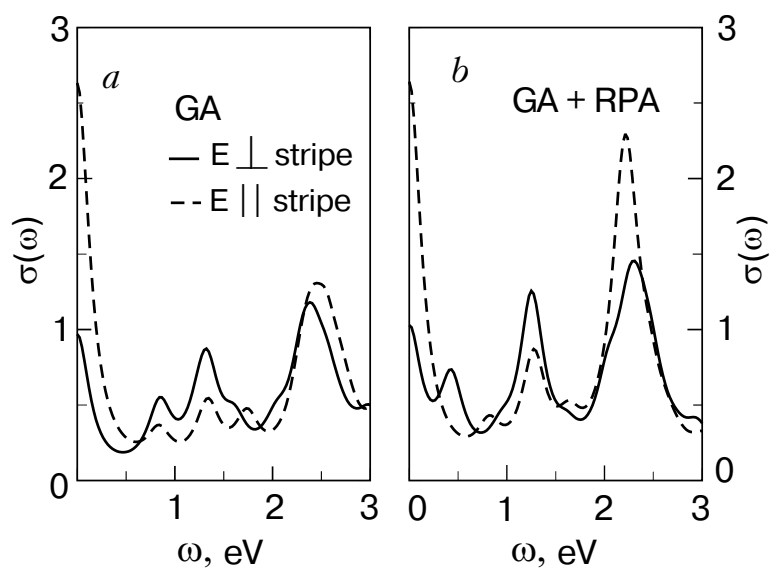

Fig. 7. Optical conductivity for $d=4$ stripes with the electric field applied perpendicular and parallel to the stripes. $\sigma(\omega)$ computed without the addition of RPA corrections. The corresponding excitations are also indicated in the bandstructure in Fig. $8(a) ; \sigma(\omega)$ computed within the GA + RPA approach $(b)$. scopic explanation of the HEMIR existed before our work [66].

Finally we note that also in isostructural $\mathrm{La}_{2} \mathrm{NiO}_{4.133}$ MIR features were related to the formation of stripes [106].

We compute the optical conductivity $\sigma(\omega)$ based on the stripe structures obtained within the three-band model in Sec. 3. However, at very dilute doping $(x \lesssim 0.03)$ due to the long-range Coulomb interaction (not included in our calculations) each hole will be close to an acceptor preventing the formation of stripes. In this case the lowest energy one-hole solution consists of a self-trapped state similar to the Zhang-Rice state [107] as found in HF [96,108]. As doping increases, the donor potential becomes more uniform and screened, favoring the formation of stripes. Experiment shows that stripes are parallel to the $\mathrm{Cu}-\mathrm{O}$ bond except at dopings $0.03 \lesssim 0.05$ where diagonal stripes have been observed [20]. Those may be an intermediate state between the isolated polarons and the vertical stripes and probably also require long-range Coulomb and (or) electron lattice interactions to be stable. For simplicity we skip this phase and consider vertical metallic solutions. Weak one-dimensional instabilities probably relevant at low temperatures are intentionally suppressed in our clusters due to finite size effects.

For inhomogeneous textures the optical conductivity depends on the direction $\alpha$ of the applied electric field and can be computed within the GA + RPA approach from

$\sigma_{\alpha}^{\prime}(\omega)=D_{\alpha} \delta(\omega)+\pi \sum_{n>0} \frac{\left|\left\langle n\left|j_{\alpha}^{G A}\right| 0\right\rangle\right|^{2}}{E_{n}-E_{0}} \delta\left[\omega-\left(E_{n}-E_{0}\right)\right]$.

Here $j^{G A_{\alpha}}$ denotes the GA current operator which contains the Gutzwiller hopping renormalization factors. The Drude weight is obtained from the $f$-sum rule

$$
\int_{0}^{\infty} d \omega \sigma_{\alpha}^{\prime}(\omega)=-\frac{1}{2} \pi\left\langle T_{\alpha}^{G A}\right\rangle
$$

which relates the integrated weight of $\sigma_{\alpha}^{\prime}(\omega)$ to the GA kinetic energy in the respective directions.

In the left (right) panel of Fig. 7 we show $\sigma(\omega)$ for both orientations of the applied electric field without (with) the addition of RPA corrections. The excitations shown in Fig. 7, $a$ can be directly obtained from the symmetry of the mean-field bands (Fig. 8) the symmetry of which determines the selection rules for optical transitions. Roughly speaking the flat bands labeled $S$ and $P$ correspond to symmetric $(S)$ and antisymmetric $(P)$ combinations of orbitals centered on the two legs of $\mathrm{Cu}$ that form the core of the stripe 


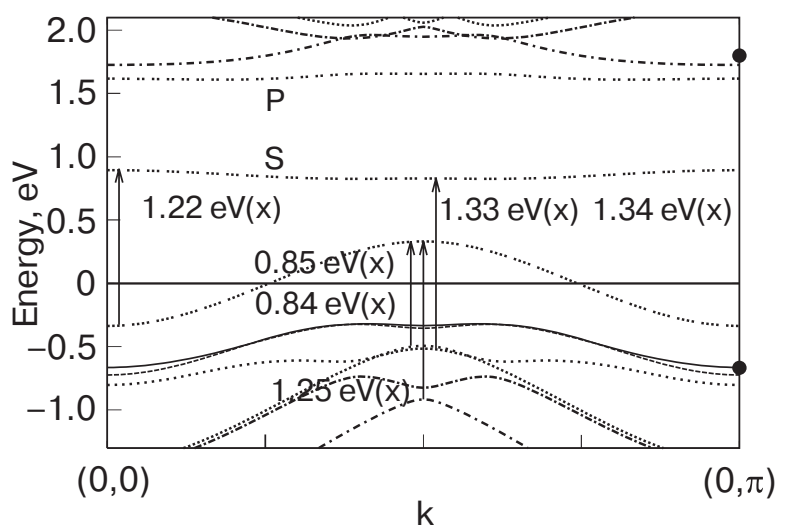

Fig. 8. Electron mean-field bands measured from the chemical potential for the momentum in the direction of the stripe $(d=7, x=0.071)$. The arrows are the lowest energy dipole allowed mean-field transitions in a $d \times 4$ cluster labeled by energy and polarization. Notice that the $x$ polarization is perpendicular to the stripe. We also plot the energies of the AF insulating bands at momentum $\mathbf{k}=(0, \pi)$ (full dots) measured from the same reference energy.

(sites 2 and 3 in Fig. 10). The band crossing the chemical potential (hereafter the «active band») is due to the orbitals centered on the core $O$ leg of the stripe (at 2.5 in Fig. 10,a). The antisymmetric $\mathrm{Cu}$ orbital combination mixes with the core $O$ orbital pushing upwards (downwards) the $P$ band (active band) close to the edge of the stoichiometric bands (marked by the full dots). The lower bands are of mainly $O$ character. All optical transitions in the $x$ direction indicated in Fig. 8 are of course between even and odd states with respect to the stripe central axis. From Fig. 7, $a$ it turns out that besides the $\mathrm{CT}$ excitation the transitions at $\omega \approx 1.3 \mathrm{eV}$ and $\omega \approx 0.85 \mathrm{eV}$ have the strongest weight in both directions of the applied electric field. The other transitions reported in Fig. 8 have much smaller spectral weights. Upon adding RPA corrections in the computation of the optical conductivity (cf. Fig. $7, b$ ) the $0.85 \mathrm{eV}(x)$ transition which has a strong oscillator strength in mean-field does not show up and instead the low-energy collective mode appears at $\sim 0.4 \mathrm{eV}$ which is also polarized perpendicular to the stripes. A similar mode was found in a study of stripes within the $t-t^{\prime}-t^{\prime \prime}-J$ model [109]. On the other hand the transitions at $\omega \approx 1.3 \mathrm{eV}$ are almost unchanged by the RPA and are only slightly enhanced in intensity. This negligible renormalization of the mean field transitions by the RPA is characteristic for incoherent particle-hole excitations whereas the mode at $0.4 \mathrm{eV}$ which shows only in the RPA is clearly a collective mode.

In order to compare with experiment one has to average the optical conductivity over the two stripe orientations with respect to the applied electric field.

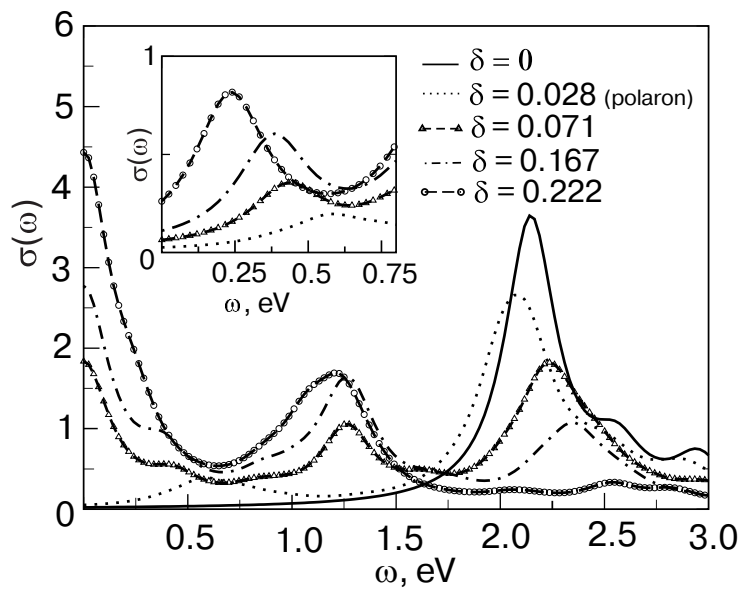

Fig. 9. Optical conductivity labeled by doping, system size and, in the case of stripes, interstripe distance. The units of conductivity are given by $\sigma_{0}=3.6 \cdot 10^{2}(\Omega \cdot \mathrm{cm})^{-1}$ with a background dielectric constant $\varepsilon_{b}=2.6$ (see Ref. 96 and caption of Fig. 12). The curve labeled $x=0.028$ corresponds to the single-hole solution. For larger dopings the figure is an average over the electric field directions parallel and perpendicular to the $\mathrm{BC}$ stripes. The inset shows the low energy spectra excluding the Drude component. We used a Lorentzian broadening of $0.2 \mathrm{eV}$.

The resulting spectra are shown in Fig. 9 for various dopings. The RPA optical conductivity for the single-hole solution appropriate at low doping, as discussed above, reproduces the results of HF + RPA [96]. Formation of a doping induced MIR band close to $0.5 \mathrm{eV}$ and doping induced transfer of spectral weight from the charge transfer band to the MIR region in agreement with experiment in this doping range [67].

For distant stripes $(d=7)$ the single-hole MIR band now splits into two bands. The one at higher energy is the band of incoherent particle-hole excitations, identified above close to $1.3 \mathrm{eV}$, and provides a theoretical explanation for the HEMIR. The position of this band is nearly independent of doping and can be understood in terms of transitions within the stripe band structure at mean-field level shown in Fig. 8. Indeed the HEMIR is mainly formed by the $1.33 \mathrm{eV}(x)$ and $1.34 \mathrm{eV}(y)$ mean field transitions with similar oscillation strengths. The other MIR band, shown also in the inset of Fig. 9, is a low-energy collective mode and has no mean-field counterpart as explained above. To characterize the LEMIR band we compute the socalled transition charges and transition currents

$$
\begin{aligned}
n_{i}^{\mu} & \equiv\left\langle 0\left|\hat{n}_{i}\right| \mu\right\rangle, \\
j_{n m}^{\mu} & \equiv\left\langle 0\left|\hat{j}_{n m}^{G A}\right| \mu\right\rangle
\end{aligned}
$$

where $\mu$ labels RPA excitations. $n_{i}^{v}\left(j_{n m}^{v}\right)$ is proportional to the time-dependent charge (current) fluctu- 


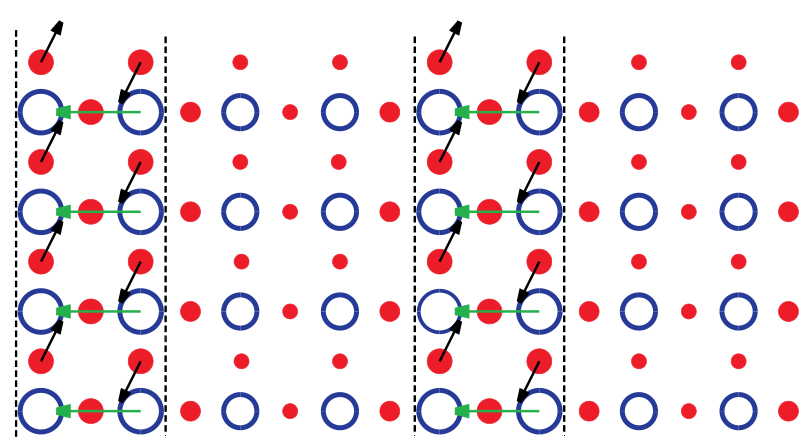

Fig. 10. Sketch of transition charges (black arrows) and transition currents (gray arrows) for a $d=4$ BC stripe (indicated by the dashed lines) when the collective MIR mode is excited. The transition charges provide a snapshot of the charge oscillation in the collective mode. The black arrows indicate charge increase (up) or decrease (down). The horizontal arrows indicate the associated current fluctuation. $\mathrm{Cu}$ and $\mathrm{O}$ sites are symbolized by open and full circles respectively.

ation $\delta n_{i}\left(\delta j_{n m}\right)$ that would occur at frequency $\omega_{v}$ if the state $v$ were weakly excited [86].

In Fig. 10 we show the charge and current fluctuations associated with the LEMIR mode. It is associated with currents flowing across the stripe enhancing (depleting) the charge density at the boundaries to the $\mathrm{AF}$ regions and thus corresponds to lateral displacements of the stripe. In fact, if we approximate the charge modulation by $\cos \left(q_{C D W} r+\theta\right)$ with $q_{C D W}=2 \pi(2 \varepsilon, 0)$, this oscillation can be interpreted as a time-dependent fluctuation of the phase $\theta$ and thus the LEMIR excitation can be identified with a phason. Optically active phasons have zero momentum, but naturally a band of phasons exist with a well-defined dispersion relation. In continuum models phasons are massless Goldstone modes whereas here the commensurability of $q_{C D W}$ with the lattice makes

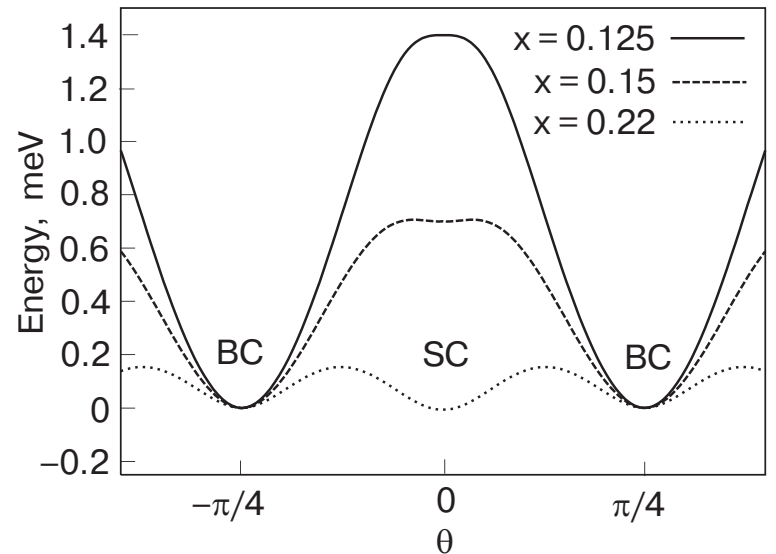

Fig. 11. Mean field energy per stripe cell (8 Cu's) as a function of the collective phase $\theta$ of the CDW for various doping levels and for $d=4$ stripes in a $4 d \times 12$ system. them have a finite energy at zero momentum. This energy however is small and decreases as doping increases. Since the BC and SC state become quasidegenerate at a doping $x_{0}$ it is natural to expect that the phason softening is related to the quasidegeneracy between these states.

In order to substantiate this idea we compute the energy landscape for «intermediate» solutions constrained to be stripes centered between $\mathrm{Cu}$ and $\mathrm{O}$. For each intermediate state we perform a Fourier analysis and extract the phase $\theta$ of the first Fourier component of the charge-density-wave (CDW) modulation. Fig. 11 shows the energy for $d=4$ stripes and different dopings. This provides an upper bound for the energy along the path connecting BC $(\theta=\pi / 4)$ and SC $(\theta=0)$ solutions where $\theta$ plays the role of a collective coordinate. The curves are periodic in $\theta$ with period $2 \pi / d$ corresponding to a translation by one elementary unit cell. Remarkably the curve acquires an extra periodicity close to optimum doping corresponding to the previously found quasidegeneracy between $\mathrm{SC}$ and $\mathrm{BC}$ solutions [33,39,47].

RPA is essentially an harmonic approximation of the energy landscape around the mean field solution ( $\mathrm{SC}$ or BC). The energy squared of each RPA mode is proportional to the curvature (or «stiffness») of the corresponding parabolic energy approximation when the system is displaced from the stationary state in the direction of the mode eigenvector (in our case parametrized by the collective coordinate $\theta$ ). Fig. 11 shows that as doping increases the stiffness decreases showing explicitly that the softening of the LEMIR feature is due to the quasidegeneracy between BC and $\mathrm{SC}$ stripes. Of course the problem is very anharmonic close to $x_{0}$ and RPA provides only a rough estimate to the phason energy. Moreover, since the barrier is strongly reduced close to $x_{0}$ we expect that anharmonic corrections will make the phason even softer and the «true» ground state will be a fluctuating mixture of $\mathrm{BC}$ and $\mathrm{SC}$ solutions. Even away from $x_{0}$ stripes show a dynamic character on a scale of a few meV [12] which is not capture by our starting-point mean-field ground state. We expect this to affect the spectra at very low energies as discussed in Ref. 102 but not at the scale of Fig. 9.

In Fig. 12 we show the optical conductivity spectral weight integrated up to an energy of $1.5 \mathrm{eV}$ $\left(N_{\text {eff }}(1.5 \mathrm{eV})\right)$ in comparison with experimental data from Ref. 67. The lower curves report the regular $(\omega>0)$ and Drude $(\omega=0)$ contributions to $N_{\text {eff }}(1.5 \mathrm{eV})$ in each direction.

For $x<1 / 8$ the number of stripes, which act as low-energy light absorbers, increases linearly with doping keeping its electronic structure practically un- 


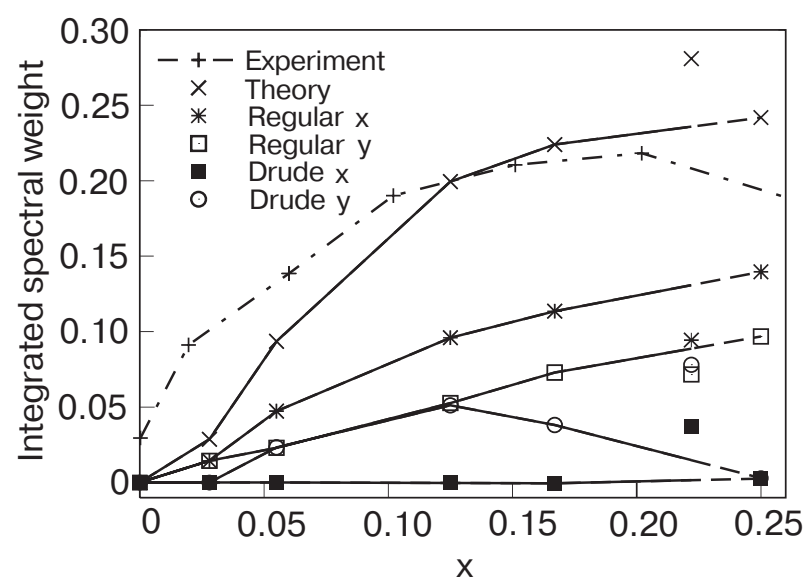

Fig. 12. Experimental [67] and theoretical integrated optical conductivity spectral weight integrated up to $1.5 \mathrm{eV}$ vs. $x$. The spectral weight is converted to an effective number of electrons $N_{\text {eff }}$ as in Ref. 67. The background dielectric constant $\varepsilon_{b}$ is our only free parameter and was adjusted to make the theoretical and experimental intensities to have an overall agreement. We also show the computed Drude and regular contributions in each direction. The dashed lines indicate the region of metastability of the $d=4$ solution. The points not joined by lines correspond to the $d=3$ solution which becomes more stable in that region.

changed [47]. Accordingly $N_{\text {eff }}(1.5 \mathrm{eV})$ increases roughly linearly with doping. For $1 / 8>x>x_{1}\left(x_{1} \sim\right.$ $\sim 0.22-0.23$ ) the number of absorbers get locked and the evolution of the spectral weight is related to changes in the electronic structure of each stripe, the more relevant one being the shift of the chemical potential from the center of the active band as discussed in Sec. 3.1 [47]. This depresses the Drude weight in the stripe direction (joined circles) and results in a slower increase of spectral weight with doping which correlates well with experiment.

For $x>x_{1} d=3$ stripes become the mean-field ground state. These solutions tend to have a larger spectral weight due to the strong Drude weight in both directions (unjoined points in Fig. 12). The nature of the ground state however is not clear in this overdoped regime and therefore our results become more qualitative than quantitative. Indeed $d=3$ stripes have not been observed in LSCO. As already mentioned one possible scenario is phase separation between the stripe solutions and the homogeneous Fermi liquid.

\section{Stripes in the one-band Hubbard model}

In Sec. 3 we have shown that within the GA applied to the three-band Hubbard model one can obtain the doping dependence of stripe textures in agreement with experiment. In principle the three-band Hamil- tonian could therefore also serve as a starting point for the investigation of magnetic excitations. However, since in this context wave-vector dependent properties are essential one has to consider large systems which makes a one-band model more appropriate for numerical reasons. Moreover, because we restrict to low-energy magnetic excitations, we do not expect that interband effects will play an important role and therefore a one-band description should in fact be sufficient. On the other hand there are small differences on the phase diagram of stable stripes solutions among the two models. We will discuss the mean-field solutions in the next section.

\subsection{Stability of stripes and parameter set}

Our investigations are based on the one-band Hubbard model with hopping restricted to nearest $(\sim t)$ and next nearest $\left(\sim t^{\prime}\right)$ neighbors

$$
H=-t \sum_{\langle i j\rangle, \sigma} c_{i, \sigma}^{\dagger} c_{j, \sigma}-t^{\prime} \sum_{\langle\langle i j\rangle\rangle, \sigma} c_{i, \sigma}^{\dagger} c_{j, \sigma}+U \sum_{i} n_{i, \uparrow} n_{i, \downarrow}
$$

Here $c_{i, \sigma}^{(\dagger)}$ destroys (creates) an electron with spin $\sigma$ at site $i$, and $n_{i, \sigma}=c_{i, \sigma}^{\dagger} c_{i, \sigma} . U$ is the on-site Hubbard repulsion.

As usual we start by finding the saddle point solutions [91]. We find that in this model and with realistic parameters (to be discussed below) the more favorable solutions are again partially filled stripes oriented parallel to the $\mathrm{Cu}_{-}^{-O}$ bond. However contrary to the three band model we find that the more favorable solutions at small doping are SC instead of BC but with a difference in energy that is negligibly small in large systems. This quasidegeneracy is also found in other one-band calculations $[33,38,110]$. Apart from this difference other features are very similar to the results of the three band model. Stripes have a width of 4, 5 lattice sites and repeating the computations of Sec. 3 one finds a magnetic incommensurability $\varepsilon=x$ for $x<1 / 8$ and $\varepsilon=1 / 8$ for $x>1 / 8$ as found experimentally [90].

We discuss now how the parameter set was determined. For definiteness in the following we mainly restrict ourself to the $\mathrm{BC}$ case because these textures constitute the more stable configuration at $x=1 / 8$ in the more accurate three-band model (cf. Sec. 3) [42] and in first principle computations [111]. However, one should keep in mind that all energetic considerations below hold equally well for SC stripes.

In Ref. 91 we have shown that the ratio $t^{\prime} / t$ is the crucial parameter which determines the filling $v$ of the stripe and therefore the agreement with the experimentally determined incommensurability [5,12]. It thus plays the same role than the oxygen-oxygen hopping $t_{p p}$ within the three-band model of Sec. 3. Inter- 


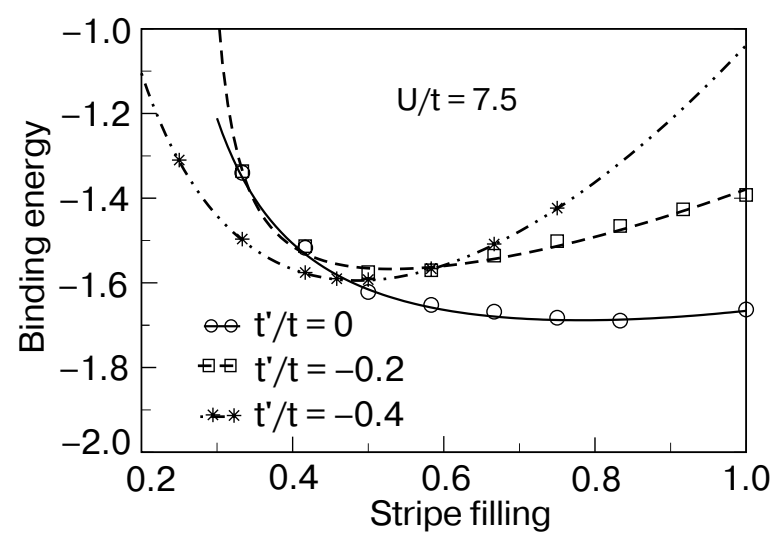

Fig. 13. $e_{h}$ (Eq. (28)) vs. filling fraction for $d=6 \mathrm{BC}$ stripes for different values of the next-nearest neighbor hopping $t^{\prime} / t$. Symbols: calculated energy on a $24 \times 12$ lattice; solid lines: fits as described in the text.

estingly it has been proposed recently [112] that a one-band model description of the various cuprate families essentially differs in the ratio between next-nearest and nearest neighbor hopping $t^{\prime} / t$. In fact it is found that the transition temperature scales with $t^{\prime} / t$ ranging from $t^{\prime} / t \approx-0.15$ for the LSCO single layer compound up to $t^{\prime} / t \approx-0.4$ for the $\mathrm{Tl}$ and $\mathrm{Hg}$ based materials. We show that this parameter plays also a key role for the stability and electronic structure of the stripes providing a possible link between stripes and superconductivity.

Figure 13 reports the energy per added hole (defined in Eq. (22) of Sec. 3) of $d=6$ BC stripes for different values of $t^{\prime} / t$ and $U / t=7.5$.

The solid lines in Fig. 13 are obtained from an expansion of the excess energy per unit length in a Taylor series in $v$ :

$$
\frac{E\left(N_{h}\right)-E_{A F}}{L}=A+B v+C v^{2} .
$$

The resulting binding energy per doped hole

$$
e_{h}=A / v+B+C v
$$

provides an excellent fit to the data points of our cluster calculation.

The parameter $A$ represents the energy to create an empty domain wall. Since this is related to an excitation of the undoped system we can estimate its value using the Heisenberg model with a magnetic interaction $J=4 t^{2} / U$ in the limit of $U \gg t$. The estimate is easily done in the case of $\mathrm{BC}$ stripes which have a core with ferromagnetic alignment along the domain wall. In the Néel limit one obtains: $A=J / 2$. The parameter $B$ can be defined as the chemical potential to add one hole into the «empty» stripe. Finally the parameter $C=C_{K}+C_{I}$ is essentially the inverse compressibility of the stripe (at fixed $d$ ) and can be split in a kinetic
$\left(C_{K}\right)$ and interaction $\left(C_{I}\right)$ contribution. $C_{K}$ can be estimated as the bandwidth of the active stripe band and is the only quantity which depends significantly on $t^{\prime}$ [91]. Since the minimum of $e_{h}$ is given by

$$
v_{\min }=\sqrt{A / C}
$$

a larger $\left|t^{\prime}\right|$ therefore implies an enhancement of the kinetic contribution $(\sim C)$ respect to the magnetic contribution $A$, and thus a shift of the minimum stripe filling to smaller values. From Fig. 13 we find that in case of $U / t=7.5$ a value of $t^{\prime} / t=-0.2$ is required in order to have $v_{\min }=0.5$ which is close to a first principle estimate [112]. The same value of $t^{\prime} / t$ has also been derived for large $U / t=10$ in Ref. 90 with the difference that in this case much smaller fillings $v_{\min }$ can be achieved for $t^{\prime} / t<<-0.2$.

The influence of a next-nearest neighbor hopping term on the stripe formation has been previously investigated by various methods. From the DMRG approach applied to the $t-t^{\prime}-J$ model [113] it turned out that a too negative $t^{\prime} / t$ can suppress both the formation of stripes and pairing correlations. A weakening of stripe tendencies for $t^{\prime} / t<0$ in the same model was also found with exact diagonalization [35] and in the Hubbard model with DMFT [114], and the HF approximation [115-117]. All these calculations suggest that static stripes are destabilized when the ratio $t^{\prime} / t$ becomes large and negative. This may indicate a more dynamical character of stripes in some systems like $\mathrm{Tl}$ and $\mathrm{Hg}$ based compounds.

The reason why we have chosen an onsite repulsion $U / t=7.5$ in the calculation of the binding energies Fig. 13 is due to the fact that this value correctly reproduces the observed energy splitting between magnons at wave-vectors $(1 / 2,0)$ and $(1 / 4,1 / 4)$ for $x=0$ [118]. Indeed, within spin-wave theory applied

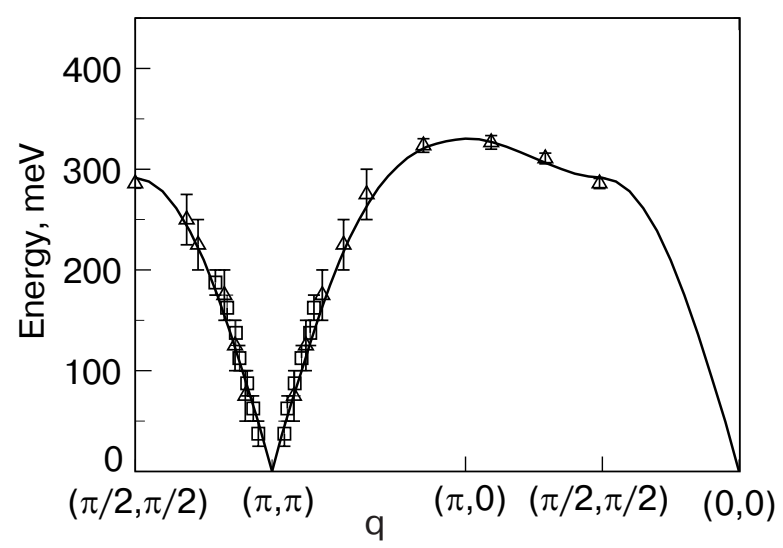

Fig. 14. Energy and wave vector dependence of magnetic excitations in the half-filled system as obtained from the $\mathrm{GA}+\mathrm{RPA}$ approach for $U / t=7.5$ and $t^{\prime} / t=-0.2$. Squares and triangles correspond to data points from INS experiments on $\mathrm{LaCuO}_{4}$ by Coldea et al. [118]. 
to the Heisenberg model (corresponding to $U / t \rightarrow \infty$ ) the magnon excitations are given by

$$
\hbar \omega_{q}=\sim \sqrt{1-\left[\cos q_{x}+\cos q_{y}\right]^{2} / 4}
$$

and thus the dispersion vanishes along the magnetic Brillouin zone. It has been argued that in cuprates corrections to the Heisenberg model arising as higher orders in a $t / U$ expansion are relevant [118-122]. The most important of such corrections is a term which cyclically exchanges four spins on a plaquette. A sizable value for this term has been revealed by analyzing phonon-assisted multimagnon infrared absorption [122] and the dispersion relation [118] shown in Fig. 14. In particular the dispersion in the $(\pi, 0)$ and $(\pi / 2, \pi / 2)$ is mainly due to this term. Since the dispersion has its origin in the finiteness of $t / U$ it should show up in the transverse excitations of the Hubbard model. The computation done in GA + + RPA is also shown in Fig. 14. One obtains a very good fit of the dispersion and this provides an accurate way to estimate the strength of the repulsion. We find $U / t=7.5$ in good agreement with other estimates [118]. The dispersion turns out to be quite insensitive to the value of $t^{\prime}$ which has been discussed above.

\subsection{Spin excitations of stripes}

Since the discovery of cuprate superconductors the elucidation of their magnetic properties has been a subject of intense research in the high- $T_{c}$ community due to their possible relevance for the superconducting mechanism [123]. However, no consensus has been reached yet wether the magnetic excitation spectra in the various cuprate materials can be traced back to some universal phenomenology which could be expected in the face of the robust nature of superconductivity. The insulating parent compounds show long-range antiferromagnetic (AF) order in the $\mathrm{CuO}_{2}$ planes below the Néel temperature [124]. This static AF order is lost above a concentration of added holes per planar copper $x \approx 0.02$ but complex dynamical spin correlations persist up to the overdoped regime [12,123].

In $\mathrm{YBa}_{2} \mathrm{Cu}_{3} \mathrm{O}_{6+y}$ (YBCO) incommensurate magnetic fluctuations have been detected by inelastic NS (INS) $[14,18,125,126]$ with a similar doping dependence of the low-energy incommensurability to that of LCO [18]. Upon increasing energy the incommensurate branches continuously disperse towards the so-called resonance mode at wave-vector $Q_{A F}=(\pi, \pi)$, a collective magnetic mode that grows up below $T_{c}$ [127-129]. The energy $E_{r}$ of the spin resonance seems to scale linearly with $T_{c}$ which has led to speculations that it could be related to the superconducting pairing and phase coherence (cf. Ref. 130,131] and references therein), thus suggesting a magnetic origin of the high transition temperatures of cuprates. Finally above $E_{r}$ the magnetic fluctuations in YBCO acquire again an incommensurate structure $[14,15,19,125,131]$ and are already observed above $T_{c}$ [126].

Very recent experiments were dedicated to explore the problem of universality in the magnetic excitations between different cuprate families [70,132-134]. In particular Tranquada and collaborators [70] reported INS measurements of the magnetic excitations in $\mathrm{La}_{1.875} \mathrm{Ba}_{0.125} \mathrm{CuO}_{4}$ which shows static charge and spin order. Most interestingly the dispersion of spin excitations shows features which resemble closely those of YBCO although the measurement has been performed above $T_{c}$. The similarity between both compounds has been further demonstrated by a high energy study of YBCO [126] and a high resolution experiment on optimally doped LCO [133]. These works [70,126,132-134] are certainly an important step towards a unified understanding of magnetic fluctuation in cuprates. On the other hand they open new questions. The spin excitations in YBCO are commonly explained in terms of an itinerant picture as arising from a dispersing two-particle bound state induced by $\mathrm{AF}$ correlations in a $d$-wave superconducting system $[134,135]$. On the other hand magnetic excitations on top of stripes are usually described in a localized moment picture within spin-wave theory (LSWT) $[136,137]$. If the origin of magnetic excitations in cuprates is universal, what picture is more appropriate? The spectra of LSWT are difficult to reconcile with several features of YBCO [126,130,134] and LCO $[70,133]$ challenging the stripe interpretation itself $[132,133]$. We have shown that the magnetic excitations in $\mathrm{La}_{1.87} \mathrm{Ba}_{0.125} \mathrm{CuO}_{4}$ (and possibly in the other cuprates) can be understood in terms of the spin fluctuations of metallic stripes which are in an intermediate regime, i.e. neither the localized nor the itinerant picture applies $[69,138]$. Contrary to the LSWT computations [136] our results are in agreement with experiment [70] over the whole range of energies and momenta.

Results shown below are obtained for lattices of dimension $\sim 100 \times 100$ sites and for doping $x=1 / 8$ corresponding to a period of charge modulation $d=4 a$.

The calculation is performed within the extended one-band Hubbard model for the parameter set derived in the pervious section. For both $\mathrm{BC}$ and $\mathrm{SC}$ stripes the magnetic excitation spectra are rather similar. For definiteness we mainly restrict ourselves to the $\mathrm{BC}$ case because of marginally better agreement with experiment (see below). As already mentioned, these textures constitute the more stable configuration 
at $x=1 / 8$ in the more accurate three-band model [42] (Sec. 3) and in first principle computations [110].

We compute the transverse dynamical structure factor.

$$
S^{\perp}(\omega, \mathbf{q})=\frac{\left(g \mu_{B}\right)^{2} \eta Z_{\chi}}{N \hbar} \sum_{v}\left|\left\langle 0\left|S_{\mathbf{q}}^{+}\right| v\right\rangle\right|^{2} \delta\left(\omega-\omega_{v}\right)
$$

which is probed by INS. Here $g=2$ and as in LSWT we included the renormalization factor $Z_{\chi}$ [124]. In the insulating phase our spectral weights are close to LSWT and therefore we adopt $Z_{\chi}=0.51$ [118]. $\eta$ takes into account polarization factors in the NS cross section and is discussed in Ref. 139. Energy is sampled at intervals of $\sim 2 \mathrm{meV}$.

Fig. 15 shows the dispersion of magnetic excitations for $\mathrm{BC}$ stripes oriented along the $y$-axis. The lower (acoustic) branch perpendicular to the stripes (b) is similar to the lowest branch found within LSWT [136]. Indeed, since the acoustic branch involves long-wavelength excitations it should not depend on the short-range details of the model. It shows the correct Goldstone-like behavior going to zero frequency at the ordering wave-vector $Q_{s}=(\pi \pm 2 \pi \varepsilon, \pi)$ with $\varepsilon=1 / 8$. Starting from $Q_{s}$ one observes two branches of spin-waves where the one dispersing towards smaller $q_{x}$ rapidly loses intensity. The other one remains very intense up to $Q_{A F}$ where it can be associated with the resonance peak. Moreover, the dispersion develops a local maximum at $Q_{A F}$ explaining the strong intensity in the momentum integrated structure factor (Fig. 16) at the resonance frequency. In the direction of the stripe the excitations display a

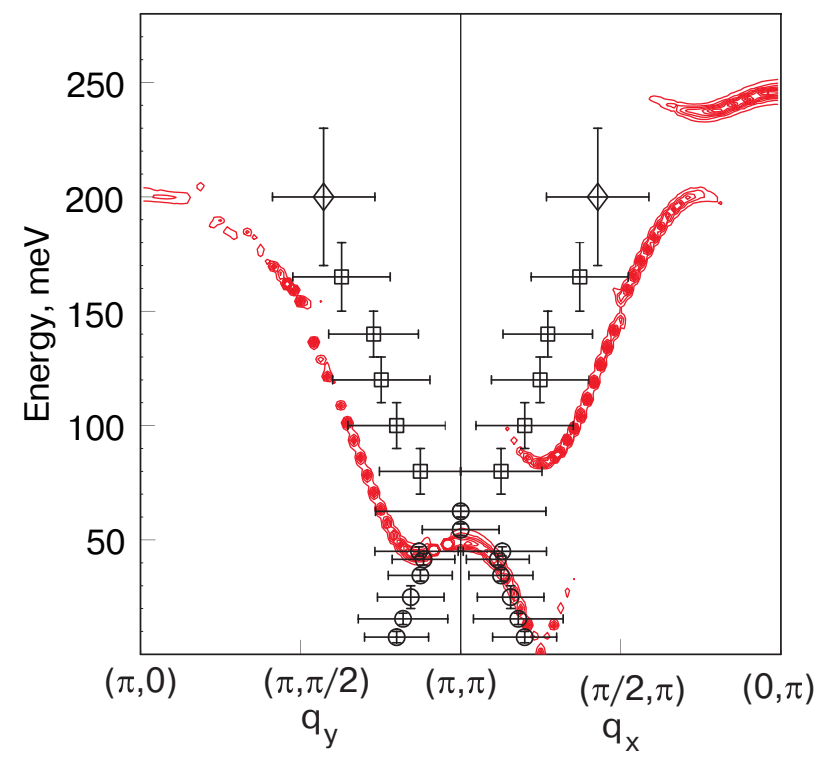

Fig. 15. Dispersion of magnetic excitations along (left) and perpendicular (right) to an array of BC stripes. We also show the experimental data from Ref. 70 .

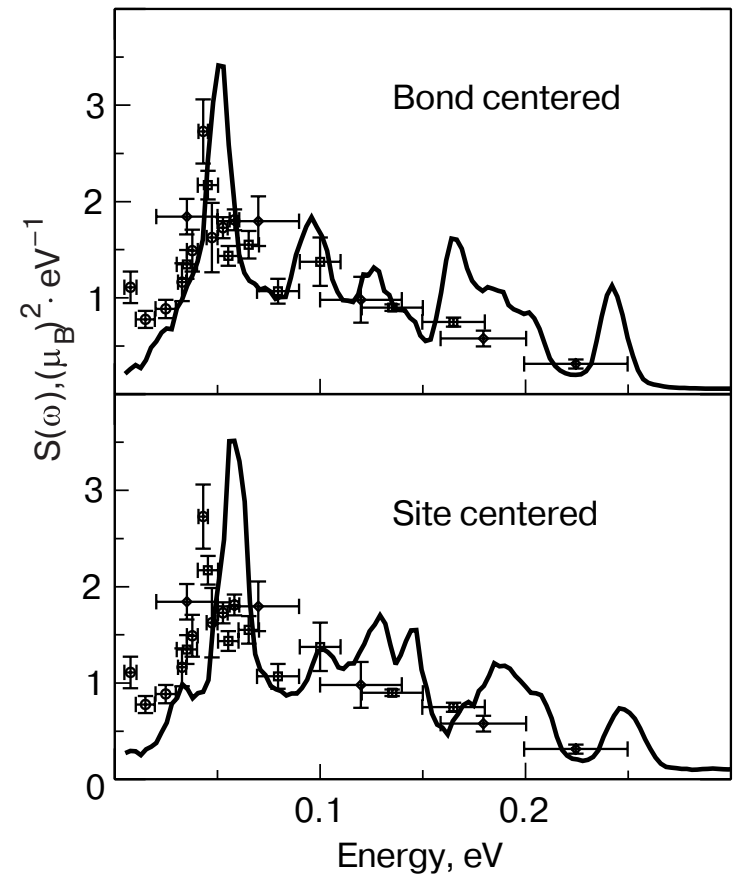

Fig. 16. $S(\omega, \mathbf{q})$ integrated on the $\mathrm{AF}$ magnetic Brillouin [140] for BC and SC stripes together with the experimental data [70]. We have fixed the polarization and intensity renormalization factor as $\eta Z_{\chi}=0.25$. For details see Ref. 139 . For the meaning of «error» bars see Ref. 70 .

«roton-like» minimum. The energy of the resonance at $Q_{A F}$ is $E_{r}=56 \mathrm{meV}$ for BC stripes which is in excellent agreement with the experimental one for this system [70]. The corresponding value for SC textures is slightly higher $\left(E_{r}=64 \mathrm{meV}\right)$.

In order to compare with experiments one should average over the two possible orientations of the stripes. In Fig. 15 this amounts to adding to each of the panels the data of the other panel reflected with respect to the central axes. One obtains an X-shaped dispersion providing a natural explanation for the $\mathrm{X}$-like feature seen in both YBCO $[14,19,134]$ and LCO [70].

The acoustic branch and its continuation in Fig. 15 are quite similar to the dispersion obtained in a weakly coupled two-leg ladder system [141]. In these approaches parameters are adjusted to drive the system into the quantum critical point separating the quantum paramagnet from the magnetically ordered state [141]. Hence the similarity with our spectra is reasonable since regarding the magnetism the ordered state corresponds to the present ground state and one can expect continuity of the excitations at the transition. The spin-leg ladder theories [142], however, rely heavily on the fine tuning of the coupling parameter between the legs and an even charge periodicity of the stripes (like for $\varepsilon=1 / 8$ ). In contrast we obtain qualitatively similar spectra for $\varepsilon=1 / 8$ and $\varepsilon=1 / 10$ 
$(x=0.1, d=5 a)$ [42,91], in accord with experiment [133]. Note that this does not exclude the interesting possibility that a small spin-gap opens by the ladder mechanism for $x \geq 1 / 8$ and $\varepsilon=1 / 8[111,141,142]$.

Above the acoustic branch in Fig. 15 there are three optical branches. For BC textures the lowest two almost touch at $q_{x}=\pi / 2$. Gap positions are in agreement with LSWT but the dispersion is not. Indeed the computations in Ref. 136 yield a shift of the two lower optical branches by $\pi / 4$ along $q_{x}$ with respect to our computation. Formally this difference can be traced back to the nonlocal nature of magnetic excitations in our system which shows magnetic moments considerably smaller than in the insulator, and more importantly is metallic. In LSWT only processes of the kind $S_{i}^{+} S_{j}^{-}$are allowed in the effective interaction kernel with $i$ and $j$ being close neighbors [136]. We find that not only processes with $i$ far from $j$ are important but also processes of the kind $c_{i, \uparrow}^{\dagger} c_{i^{\prime}, \downarrow} c_{j, \downarrow}^{\dagger} c_{j^{\prime}, \uparrow}$ with all four sites different.

Figure 17 reports constant frequency scans of $S^{\perp}(\omega, \mathbf{q})$ in the full Brillouin zone for BC stripes oriented along the $y$-direction. At low energies $(\omega=6 \mathrm{meV})$ intensity is confined to the momenta of the Goldstone mode $Q_{s}=( \pm 3 \pi / 4, \pm \pi)$ and develops into the structure of a spin-wave cone at higher energies $(\omega=36 \mathrm{meV})$. The intensity is very anisotropic along the intersection with the region closest to $Q_{A F}$ displaying the stronger weight. At $\omega=55 \mathrm{meV}$ the acoustic mode has reached the resonance so that main intensity is now at $Q_{A F}=(\pi, \pi)$. However, due to the appearance of the roton we also observe some weight in the direction of the stripes appearing as the small segments at $\approx( \pm \pi, \pm \pi / 4)$. Upon further increasing the energy $(\omega=80 \mathrm{meV})$ one still only observes intensity in the direction of the stripes due to the gap between acoustic mode and lowest energy optical mode perpendicular to the stripe (cf. Fig. 15). The intensity of the latter becomes visible for $\omega \geq 90 \mathrm{meV}$ and leads to the ring shaped structure around $Q_{A F}$ for higher energies.

Another very interesting finding are the horizontal cigar like features seen at $36 \mathrm{meV}$ and higher energies. Similar features have been obtained in weak coupling in the longitudinal channel [53]. These structures appear close to $q_{y} \sim \pi / 2 \sim 2 k_{F}$ and correspond to spin-flip backscattering processes of the quasi-one dimensional metallic subsystem. At this $q_{y}$ the continuum extends to about $100 \mathrm{meV}$. As expected there is also a sharp collective mode corresponding to forward scattering processes but with too weak intensity to be observable in Fig. 17. Diffusive scattering revealed a similar phenomenon in $\mathrm{La}_{5} / \mathrm{Ni}_{1 / 3} \mathrm{O}_{4}$ where stripes are insulating but have a spin-1 $/ 2$ degree of freedom at the core [143].

In order to compare with the experimentally detected intensities (shown in Fig. 2 of Ref. 70) one has to average over the two stripe orientations, i.e., to superimpose the panels in Fig. 17 with the analogous figure rotated by $90^{\circ}$. The resulting intensity scans are shown in Fig. 18 restricted to the magnetic zone only and rotated by $45^{\circ}$ to facilitate comparison with the corresponding panels of Ref. 70 .

Resolution and linewidth effects are considered by convoluting the data with a gaussian ( FWHM $=0.06$ r.l.u.). It is interesting to observe that below the resonance and due to the highly anisotropic intensity distribution around the spin-wave cone the acoustic mode now appears as a single branch dispersing towards $(\pi, \pi)$. The mode dispersing away from $(\pi, \pi)$ has much smaller intensity (c.f. Fig. 15) and is not visible in Fig. 18. Although our study focusses on an underdoped system this feature fits nicely with the fact that only the branch closest to $Q_{A F}$ is seen in a recent high resolution study on optimally doped LCO [133]. At $55 \mathrm{meV}$ the «resonance» now appears as a large intensity spot at $Q_{A F}$ and some weight around due to the appearance of the roton. At higher energies the intensity evolves in a ring shaped feature around $Q_{A F}$ where the dominant weight has now rotated towards the diagonal directions. This is in fact the behavior observed in LBCO [70] but even in strongly underdoped YBCO [126] above the resonance.

\section{Conclusions}

Strongly correlated systems with competing interactions have a natural tendency to form inhomogeneous ground states. The inhomogeneities can support new collective modes which are not present in the homogeneous phase and which can have important physical consequences. Indeed an exciting possibility is that these new collective modes are responsible for pairing in high temperature superconductors and for the anomalous normal state properties.

We have reviewed the application of the time dependent Gutzwiller approximation to compute dynamical properties of cuprates. This approach [58-60] allows to compute RPA like fluctuations on top of the GA solutions with the conceptual simplicity of a HF+ RPA computation but staring from the correct ground state.

A comparison with exact data [58-60] shows that $\mathrm{GA}+\mathrm{RPA}$ is much more accurate than $\mathrm{HF}+\mathrm{RPA}$. Roughly speaking since the GA contains ground state correlations not included in a HF wave function the residual interaction is a smaller perturbation to the MF state and hence it is natural that RPA works much better. In other words RPA fluctuations on top of a 
«correlated» mean field state (GA) converge much better to the exact result than fluctuation on top of an uncorrelated mean field state (HF).
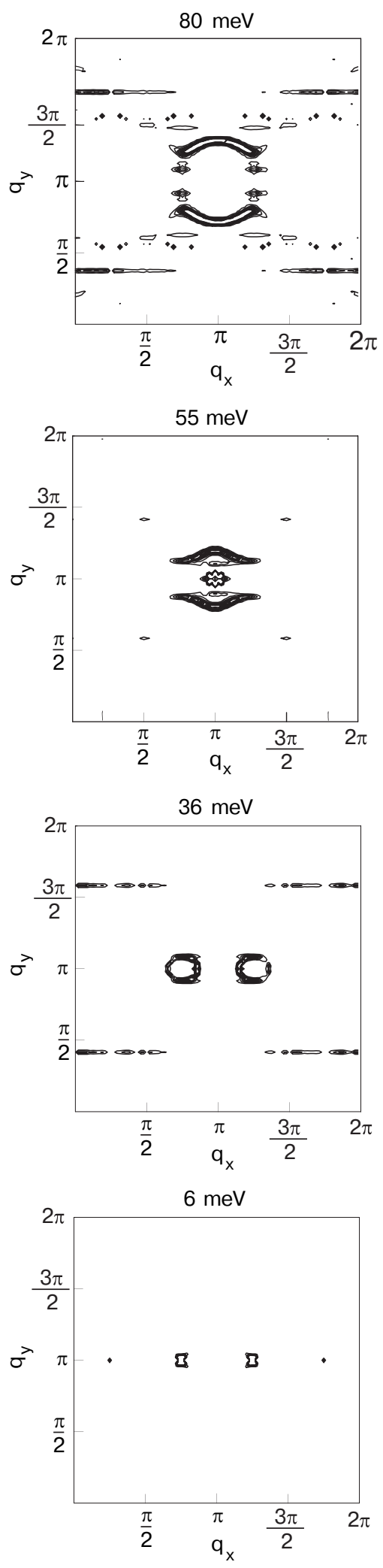

Compared to numerical «exact» methods like exact diagonalization or Monte Carlo our approach can be pushed to much larger systems. Our experience on
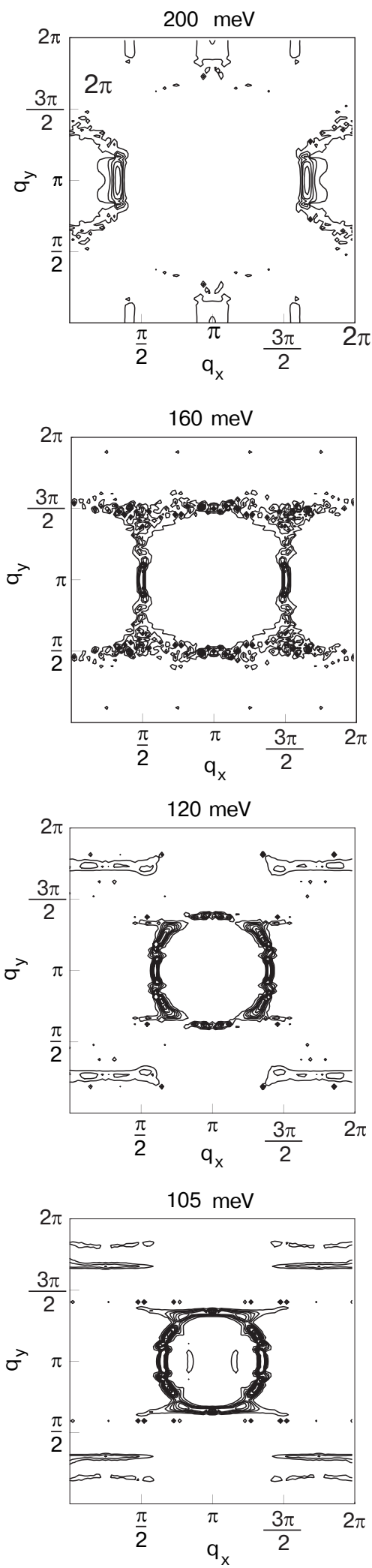

Fig. 17. Constant energy scans of $S^{\perp}(\omega, \mathbf{q})$ for BC stripes oriented along the $y$-direction. The size of the panels represent the full Brillouin zone. The title indicates the energy of the scan. The intensities are averaged in an energy window $\Delta \omega=5 \mathrm{meV}$ for energies $\omega \leq 55 \mathrm{meV}$ and $\Delta \omega=10 \mathrm{meV}$ for higher frequencies. 


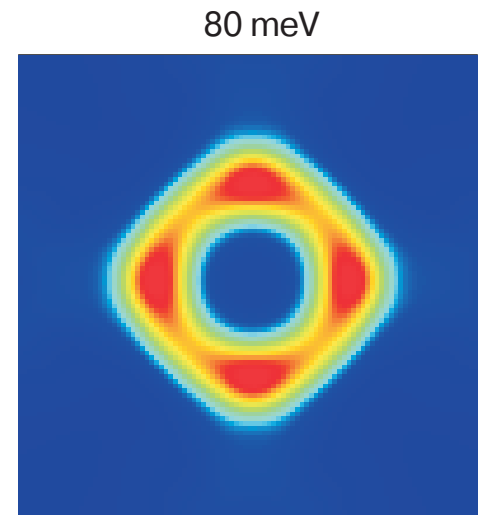

$55 \mathrm{meV}$

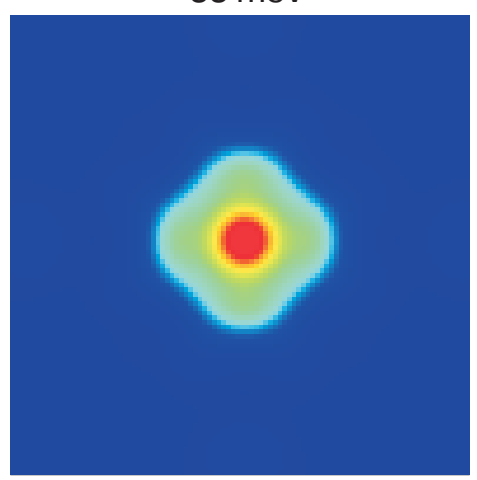

$36 \mathrm{meV}$

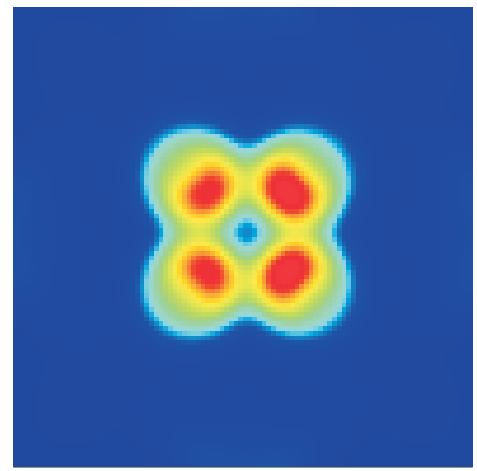

$6 \mathrm{meV}$

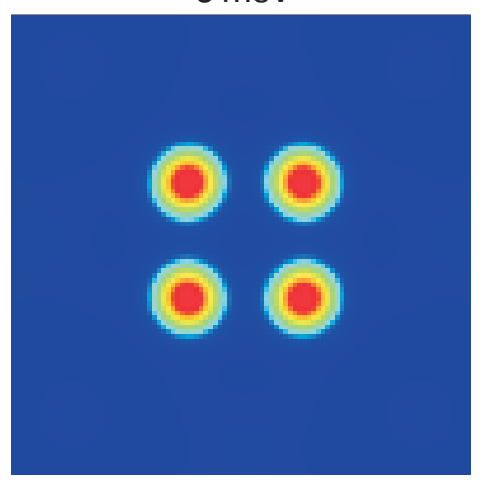

$200 \mathrm{meV}$

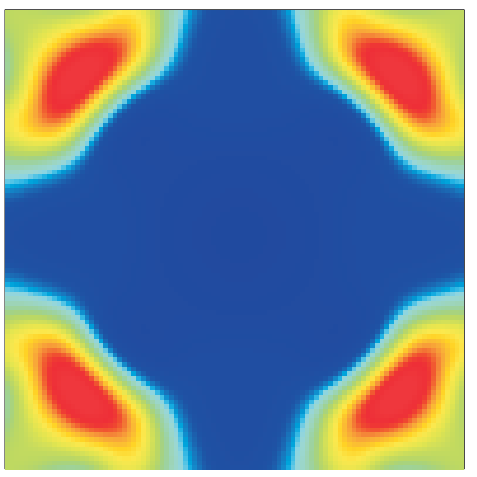

$160 \mathrm{meV}$

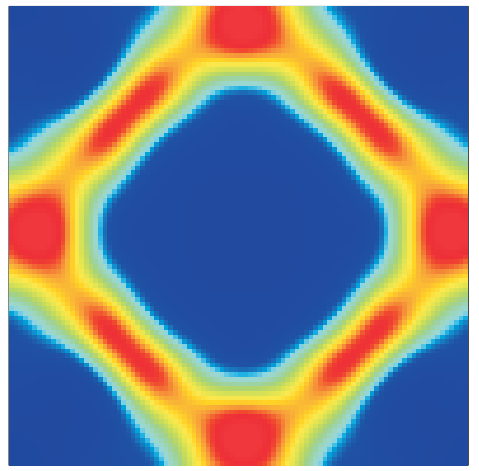

$120 \mathrm{meV}$

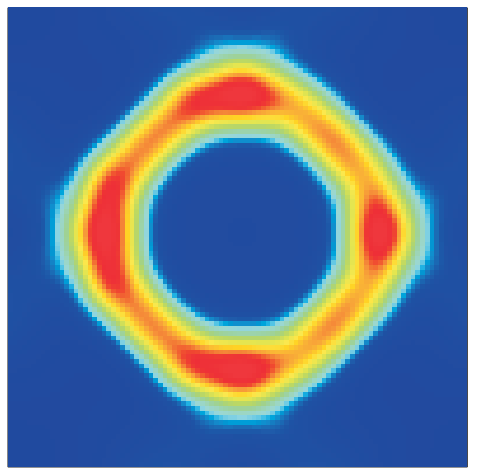

$105 \mathrm{meV}$

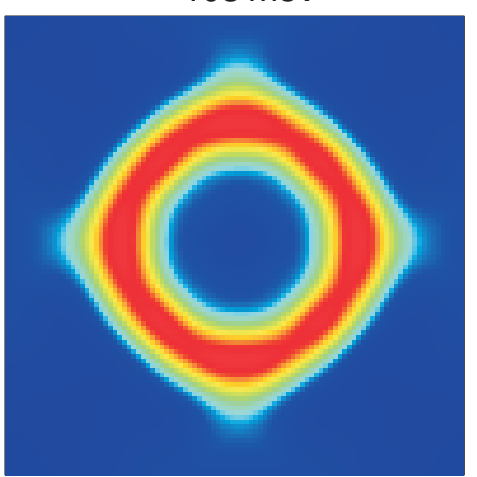

Fig. 18. Constant frequency scans of $S^{\perp}(\omega, \mathbf{q})$ for BC stripes convolved with a Gaussian (FWHM = 0.06 r.l.u.). The title indicates the energy or the energy window $(e-i)$ over which intensities are averaged. In order to compare with the experimental data of Ref. 70 the panels show the average of both horizontal and vertical stripes with the coordinate system rotated by $45^{\circ}$ with wave-vector units of $2 \pi /(\sqrt{2 a})$. 
modeling real data is that often finite size effects are more severe than the inaccuracies introduced by mean-field + RPA approaches.

A more recent approach for dynamical properties consists of mapping the problem onto quantum impurity models «dynamical mean-field theory» which, becomes exact in the limit of large dimensions. This has enormously increased our understanding of these systems, however on making the limit of large dimensions important parts of the physics are lost. For example all acoustic - like collective behavior like spin-waves disappears. On the other hand these collective effects are naturally captured in our approach.

As a first step in the present application to the cuprates we have analyzed the saddle point solutions that minimize the GA energy functional with realistic Hamiltonian parameters. Stripes in our computation are metallic charge and spin density-waves in a regime intermediate between localized moment and itinerant pictures. These solutions allow to understand several static properties of lanthanum cuprates [42] like the doping dependence of: the magnetic incommensurability, the chemical potential and the presence or absence of particle hole symmetry as reveled by transport experiments.

Clearly the GA static solutions are an excellent starting point as opposed to Hartree-Fock (HF) solutions where the stabilization of stripes requires the manipulation of the parameters beyond the physical range.

Charge excitations determine the optical conductivity which we have computed within the GA + RPA approach. Striped domain walls induce two MIR excitations. At around $1.3 \mathrm{eV}$ a HEMIR band appears which is related to interband transitions within the stripe band structure. Further on we found a collective mode (i.e., the LEMIR band) which softens as a function of doping due to the suppression of the energy barrier between quasidegenerate $\mathrm{BC}$ and $\mathrm{SC}$ stripe solutions. These features are in good agreement with experiments for positions and relative intensities with parameters fixed by first principle computations as in Ref. 47.

For the magnetic channel probed by INS experiments our results are also in good agreement with experiments in $\mathrm{La}_{1.875} \mathrm{Ba}_{0.125} \mathrm{CuO}_{4}$ providing a straightforward explanation of the energy and momentum dependent evolution of $S^{\perp}(q, \omega)$ in terms of stripes and hence confirming stripes as a robust feature of this system with a firm theoretical basis.

The fact that the magnetic excitation spectra are similar in different cuprate families and for different doping levels suggests that stripes or the proximity to stripe instabilities are a universal property of cuprates and hence that they may be relevant for the superconducting mechanism.

Both in the charge and in the spin channel the dynamics is well explained by the striped GA state without free parameters giving strong support to the stripe scenario. In addition it show that the method is simple an accurate enough to obtain realistic dynamical properties of textured strongly correlated systems.

It is worth speculating that scattering of holes moving along and perpendicular to the stripes with the soft collective modes in the charge and spin channel may be responsible for the anomalous normal state behavior in cuprates. In addition such scattering may contribute to the pairing of holes and be responsible for the phenomenon of high temperature superconductivity. More work is needed to test this hypothesis in detail.

\section{Appendix: Spin-rotational invariant GA}

Our aim is the derivation of an approximate single-particle hamiltonian which obeys spin-rotational invariance as a generalization of the conventional mean-field Kotliar-Ruckenstein Hamiltonian. For this purpose it is convenient to introduce the spinor operators

$$
\boldsymbol{\Psi}_{\mathbf{i}}^{\dagger}=\left(c_{i \uparrow}^{\dagger}, c_{i \downarrow}^{\dagger}\right) \quad \Psi_{\mathbf{i}}=\left(\begin{array}{c}
c_{i \uparrow} \\
c_{i \downarrow}
\end{array}\right),
$$

and define the spin vector components as

$$
\begin{aligned}
& S_{i}^{x}=\frac{1}{2} \boldsymbol{\Psi}_{\mathbf{i}}^{\dagger} \tau_{\mathbf{x}} \boldsymbol{\Psi}_{\mathbf{i}}=\frac{1}{2}\left(c_{i \uparrow}^{\dagger} c_{i \downarrow}+c_{i \downarrow}^{\dagger} c_{i \uparrow}\right), \\
& S_{i}^{y}=\frac{1}{2} \boldsymbol{\Psi}_{\mathbf{i}}^{\dagger} \tau_{\mathbf{y}} \boldsymbol{\Psi}_{\mathbf{i}}=\frac{1}{2}\left(c_{i \uparrow}^{\dagger} c_{i \downarrow}-c_{i \downarrow}^{\dagger} c_{i \uparrow}\right), \\
& S_{i}^{z}=\frac{1}{2} \boldsymbol{\Psi}_{\mathbf{i}}^{\dagger} \tau_{\mathbf{z}} \boldsymbol{\Psi}_{\mathbf{i}}=\frac{1}{2}\left(c_{i \uparrow}^{\dagger} c_{i \uparrow}-c_{i \downarrow}^{\dagger} c_{i \downarrow}\right) .
\end{aligned}
$$

$\tau_{i}$ denote the Pauli matrices and one can furtheron define the usual raising and lowering operators as

$$
\begin{aligned}
& S_{i}^{+}=S_{i}^{x}+i S_{i}^{y}=c_{i \uparrow}^{\dagger} c_{i \downarrow}, \\
& S_{i}^{-}=S_{i}^{x}-i S_{i}^{y}=c_{i \downarrow}^{\dagger} c_{i \uparrow} .
\end{aligned}
$$

The procedure implemented in the following consists essentially of three steps. Assume that in our initial reference frame we have non-vanishing spin order pointing locally in some given direction, i.e. $\left\langle\mathbf{S}_{i}\right\rangle \neq 0$. At first we then rotate locally to a new frame where spins point along the $z$-axis, i.e. $\left\langle\tilde{\mathbf{S}}_{i}\right\rangle=\left\langle\left(0,0, \tilde{S}_{i}^{z}\right)\right.$. This allows, as a second step, the introduction of slave-bosons and associated fermions $f_{i \sigma}$ within the Kotliar-Ruckenstein [77] scheme. For the bosons we 
apply the saddle-point (mean-field) approximation. Finally, in a third step we rotate the fermions back to the original reference frame.

We define the local rotations in spin space by the following transformations

$$
\boldsymbol{\Phi}_{\mathrm{i}}=\mathbf{U}_{\mathrm{i}}^{\dagger} \boldsymbol{\Psi}_{\mathrm{i}} \quad \boldsymbol{\Phi}_{\mathrm{i}}^{\dagger}=\boldsymbol{\Psi}_{\mathrm{i}}^{\dagger} \mathbf{U}_{\mathrm{i}}
$$

where

$$
\mathbf{U}_{\mathbf{i}}=\mathbf{1} \cos \left(\varphi_{i} / 2\right)+i \sin \left(\varphi_{i} / 2\right) \tau \boldsymbol{\eta}
$$

and $\boldsymbol{\eta}=\left(\eta_{x}, \eta_{y}, 0\right)$ is the rotation axis of length unity. The inverse transformation reads as

$$
\boldsymbol{\Psi}_{\mathrm{i}}=\mathbf{U}_{\mathrm{i}} \boldsymbol{\Phi}_{\mathrm{i}} \quad \boldsymbol{\Psi}_{\mathrm{i}}^{\dagger}=\boldsymbol{\Phi}_{\mathrm{i}}^{\dagger} \mathbf{U}_{\mathrm{i}}^{\dagger} .
$$

Within the first step of our procedure we have the requirement that the transformed spinor is given by $\tilde{\mathbf{S}}_{i}=\left(0,0, \tilde{S}_{i}^{z}\right)$. Applying the transformation Eq. (A.9) to this vector one obtains the following relations

$$
\begin{gathered}
S_{i}^{x}=-\eta_{y} \sin \left(\varphi_{i}\right) \tilde{S}_{i}^{z,}, \\
S_{i}^{y}=\eta_{x} \sin \left(\varphi_{i}\right) \tilde{S}_{i}^{z,}, \\
S_{i}^{z}=\cos \left(\varphi_{i}\right) \tilde{S}_{i}^{z} .
\end{gathered}
$$

Note that the local charge

$$
n_{i}=\boldsymbol{\Psi}_{\mathbf{i}}^{\dagger} \mathbf{1} \boldsymbol{\Psi}_{\mathbf{i}}=\tilde{n}_{i}
$$

and thus also the interaction term $n_{i \uparrow} n_{i \downarrow}=$ $=1 / 2\left(n_{i}^{2}-n_{i}\right)$ are not affected by the transformation.

Since by definition transverse spin order vanishes in the rotated frame we can now, as a second step, apply the Kotliar-Ruckenstein slave-boson scheme:

$$
\tilde{c}_{i \sigma}=z_{i \sigma} \tilde{f}_{i \sigma} \quad \tilde{c}_{i \sigma}^{\dagger}=z_{i \sigma}^{\dagger} \tilde{f}_{i \sigma}^{\dagger}
$$

with

$$
\begin{gathered}
z_{i \sigma}=\frac{1}{\sqrt{e_{i}^{\dagger} e_{i}+p_{i,-\sigma}^{\dagger} p_{i,-\sigma}}}\left[e_{i}^{\dagger} p_{i \sigma}+p_{i,-\sigma}^{\dagger} d_{i}\right] \times \\
\times \frac{1}{\sqrt{d_{i}^{\dagger} d_{i}+p_{i, \sigma}^{\dagger} p_{i, \sigma}}}
\end{gathered}
$$

The double $(d)$, singly $\left(p_{\sigma}\right)$, and empty $\left(e_{i}\right)$ occupancy bosons are constrained by the following relations:

$$
\begin{array}{r}
\sum_{\sigma} p_{i, \sigma}^{\dagger} p_{i, \sigma}+2 d_{i}^{\dagger} d_{i}=2 \tilde{n}_{i}=2 n_{i}, \quad \text { (A.16) } \\
p_{i, \uparrow}^{\dagger} p_{i, \uparrow}-p_{i, \downarrow}^{\dagger} p_{i, \downarrow}=2 \tilde{S}_{i}^{z}=2 S_{i}^{z} / \cos \left(\varphi_{i}\right),(\mathrm{A} .17)
\end{array}
$$

with

$$
\tan ^{2} \varphi_{i}=\frac{S_{i}^{+} S_{i}^{-}}{\left(S_{i}^{z}\right)^{2}}
$$


and for simplicity we have skipped the $\langle\ldots\rangle$ symbols in denoting the expectation values of the $S$ components.

We finally obtain for the spin-rotational invariant Gutzwiller energy functional Eq. (4) for the Hubbard model

$$
E^{G A}=\sum_{i, j} t_{i j}\left\langle\boldsymbol{\Psi}_{\mathbf{i}}^{\dagger} \mathbf{z}_{\mathbf{i}} \mathbf{z}_{\mathbf{j}} \boldsymbol{\Psi}_{\mathbf{j}}\right\rangle+U \sum_{i} D_{i}
$$

1. J.G. Bednorz and K.A. Müller, Z. Physik B64, 189 (1986).

2. Proceedings of the First Workshop on Phase Separation in Cuprate Superconductors, K.A. Müller and G. Benedek (eds.); World Scientific, Singapure (1993).

3. Proceedings of the Second Workshop on Phase Separation in Cuprate Superconductors, E. Sigmund and K.A. Müller (eds.); Springer Verlag, Berlin Heidelberg (1994).

4. Proceedings of the Third Workshop on Phase Separation, Electronic Inhomogeneitites and Related Mechanisms in high-T $T_{C}$ Superconductors, C.Di Castro and E. Sigmund (eds.); J. Supercond. 9 (1996).

5. J.M. Tranquada, B.J. Sternlieb, J.D. Axe, Y. Nakamura, and S. Uchida, Nature 375, 56 (1995).

6. S.M. Hayden, G.H. Lander, J. Zarestky, P.J. Brown, C. Stassis, P. Metcalf, and J.M. Honig, Phys. Rev. Lett. 68, 1061 (1992).

7. J.M. Tranquada, D.J. Buttrey, V. Sachan, and J.E. Lorenzo, Phys. Rev. Lett. 73, 1003 (1994).

8. C.H. Chen, S.W. Cheong, and A.S. Cooper, Phys. Rev. Lett. 71, 2461 (1993).

9. A. Vigliante, M. von Zimmermann, J.R. Schneider, T. Frello, N.H. Andersen, J. Madsen,D.J. Buttrey, Doon Gibbs, and J.M. Tranquada, Phys. Rev. B56, 8248 (1997).

10. J.M. Tranquada, J.D. Axe, N. Ichikawa, Y. Nakamura, S. Uchida, and B. Nachumi, Phys. Rev. B54, 7489 (1996).

11. J.M. Tranquada, J.D. Axe, N. Ichikawa, A.R. Moodenbaugh, Y. Nakamura, and S. Uchida, Phys. Rev. Lett. 78, 338 (1997).

12. K. Yamada, C.H. Lee, K. Kurahashi, J. Wada, S. Wakimoto, S. Ueki, H. Kimura, Y. Endoh, S. Hosoya, G. Shirane, R.J. Birgeneau, M. Greven, M.A. Kastner, and Y.J. Kim, Phys. Rev. B57, 6165 (1998).

13. T. Niemöller, N. Ishikawa, T. Frello, H. Hünnefeld, N.H. Andersen, S. Uchida, J.R. Schneider, and J.M. Traquada, Eur. Phys. J. B12, 509 (1999).

14. M. Arai, T. Nishijima, Y. Endoh, T. Egami, S. Tajima, K. Tomimoto, Y. Shiohara, M. Takahashi, A. Garrett, and S.M. Bennington, Phys. Rev. Lett. 83, 608 (1999)

15. M. Arai, Y. Endoh, S. Tajima, and S.M. Bennington, Int. J. Mod. Phys. B14, 3312 (2000).

16. M. Fujita, H. Goka, K. Yamada, J.M. Tranquada, and L.P. Regnault, Phys. Rev. B70, 104517 (2004).
17. A.R. Moodenbaugh, Y. Xu, M. Suenaga, T.J. Folkerts, and R.N. Shelton, Phys. Rev. B38, 4596 (1988).

18. P. Dai, H.A. Mook, R.D. Hunt, and F. Dogan, Phys. Rev. B63, 054525 (2001).

19. H.A. Mook, P. Dai, and F. Dogan, Phys. Rev. Lett. 88, 097004 (2002).

20. M. Matsuda, M. Fujita, K. Yamada, R.J. Birgeneau, M.A. Kastner, H. Hiraka, Y. Endoh, S. Wakimoto, and G. Shirane, Phys. Rev. B62, 9148 (2000).

21. M. Fujita, K. Yamada, H. Hiraka, P.H. Gehring, S.H. Lee, S. Wakimoto, and G. Shirane, Phys. Rev. B65, 064505 (2002).

22. H. He, Y. Sidis, P. Bourges, G.D. Gu, A. Ivanov, N. Koshizuka, B. Liang, C.T. Lin, L.P. Regnault, E. Schoenherr, and B. Keimer, Phys. Rev. Lett. 86, 1610 (2001)

23. H.A. Mook, F. Dogan, and B.C. Chakoumakos, cond-mat/9811100, Stripes and Related Phenomena, A. Bianconi and N.L. Saini (eds.), Kluwer Academic (2000)

24. S. Krämer and M. Mehring, Phys. Rev. Lett. 83, 396 (1999)

25. G.B. Teitel'baum, B. Büchner, and H. de Gronckel, Phys. Rev. Lett. 84, 2949 (2000).

26. P.M. Singer, A.W. Hunt, and T. Imai, Phys. Rev. Lett. 88, 047602 (2002).

27. J. Haase, C.P. Slichter, and C.T. Milling, J. Supercond. 15, 339 (2002).

28. J. Zaanen and O. Gunnarsson, Phys. Rev. B40, 7391 (1989).

29. K. Machida, Physica C158, 192 (1989).

30. H.J. Schulz, Phys. Rev. Lett. 64, 1445 (1990).

31. D. Poilblanc and T.M. Rice, Phys. Rev. B39, 9749 (1989)

32. S.W. Cheong, G. Aeppli, T.E. Mason, H. Mook, S.M. Hayden, P.C. Canfield, Z. Fisk, K.N. Clausen, and J.L. Martinez, Phys. Rev. Lett. 67, 1791 (1991).

33. S.R. White and D.J. Scalapino, Phys. Rev. Lett. 80, 1272 (1998); S.R. White and D.J. Scalapino, Phys. Rev. Lett. 81, 3227 (1998).

34. C. Stephen Hellberg and E. Manousakis, Phys. Rev. Lett. 83, 132 (1999).

35. T. Tohyama, C. Gazza, C.T. Shih, Y.C. Chen, T.K. Lee, S. Maekawa, and E. Dagotto, Phys. Rev. B59, R11649 (1999).

36. F. Becca, L. Capriotti, and S. Sorella, Phys. Rev. Lett. 87, 167005 (2001).

37. A. Himeda, T. Kato, and M. Ogata, Phys. Rev. Lett. 88, 117001 (2002).

38. M. Fleck, A.I. Lichtenstein, and E. Pavarini, Phys. Rev. Lett. 84, 4962 (2000); M. Fleck, A.I. Lichtenstein, and A.M. Ole's, Phys. Rev. B64, 134528 (2001).

39. M. Fleck, A. I. Lichtenstein, Eva Pavarini, and A. M. Ole's, Phys. Rev. Lett. 84, 4962 (2000).

40. X.J. Zhou, P. Bogdanov, S. Kellar, T. Noda, H. Eisaki, S. Uchida, Z. Hussain, and Z.X. Shen, Science 286, 268 (1999). 
41. S.R. White and D.J. Scalapino, Phys. Rev. Lett. 91, 136403, (2003).

42. J. Lorenzana and G. Seibold, Phys. Rev. Lett. 89, 136401 (2002).

43. V.J. Emery and S.A. Kivelson, Physica 209, 597 (1993).

44. U. Löw, V.J. Emery, K. Fabricius, and S.A. Kivelson, Phys. Rev. Lett. 72, 1918 (1994).

45. C. Castellani, C. Di Castro, and M. Grilli, Phys. Rev. Lett. 75, 4650 (1995).

46. J. Lorenzana, C. Castellani, and C. Di Castro, Phys. Rev. B64, 235127 (2001)

47. J. Lorenzana, C. Castellani, and C. Di Castro, Europhys. Lett. 57, 704 (2002).

48. J. Bardeen, L.N. Cooper and J.R. Schrieffer, Phys. Rev. 108, 1175 (1957).

49. E. Kaneshita, R. Morino, M. Ichioka, and K. Machida, J. Phys. Chem. Solids 63, 1545 (2002).

50. S. Varlamov and G. Seibold, Phys. Rev. B65, 075109 (2002).

51. E. Kaneshita, M. Ichioka, and K. Machida, Phys. Rev. Lett. 88, 115501 (2002).

52. M. Ichioka, E. Kaneshita, and K. Machida, J. Phys. Soc. Jpn. 70, 818 (2001).

53. E. Kaneshita, M Ichioka, and K. Machida J. Phys. Soc. Jpn, 70, 866 (2001).

54. M. Inui and P.B. Littlewood, Phys. Rev. B44, 4415 (1991).

55. M.S. Hybertsen, E.B. Stechel, W.M.C. Foulkes, and M. Schlüter, Phys. Rev. B45, 10032 (1992).

56. G. Seibold, E. Sigmund, and V. Hizhnyakov, Phys. Rev. B57, 6937 (1998).

57. A. Sadori and M. Grilli, Phys. Rev. Lett. 84, 5375 (2000).

58. G. Seibold, F. Becca, and J. Lorenzana, Phys. Rev. B67, 085108 (2003).

59. G. Seibold, F. Becca, P. Rubin, and J. Lorenzana, Phys. Rev. B69, 155113 (2004).

60. G. Seibold and J. Lorenzana, Phys. Rev. Lett. 86, 2605 (2001).

61. M.C. Gutzwiller, Phys. Rev. 137, A1726 (1965).

62. A. Ino, T. Mizokawa, A. Fujimori, K. Tamasaku, H. Eisaki, S. Uchida, T. Kimura, T. Sasagawa, and K. Kishio, Phys. Rev. Lett. 79, 2101 (1997).

63. N. Harima, J. Matsuno, A. Fujimori, Y. Onose, Y. Taguchi, and Y. Tokura, Phys. Rev. B64, R220507 (2001).

64. T. Noda, H. Eisaki and S. Uchida, Science 286, 265 (1999).

65. Yayu Wang and N.P. Ong, Proc. Natl. Acad. Sci. U.S.A 98, 11091 (2001).

66. J. Lorenzana and G. Seibold, Phys. Rev. Lett. 90, 066404 (2003).

67. S. Uchida, T. Ido, H. Takagi, T. Arima, Y. Tokura, and S. Tajima, Phys. Rev. B43, 7942 (1991).

68. M. Suzuki, Phys. Rev. B39, 2312 (1989).

69. G. Seibold and J. Lorenzana, Phys. Rev. Lett. 94, 107006 (2005).
70. J.M. Tranquada, H. Woo, T.G. Perring, H. Goka, G.D. Gu, G. Xu, M. Fujita, and K. Yamada, Nature 429, 534 (2004).

71. J. Bünemann, F. Gebhard, and W. Weber, condmat $/ 0006283$.

72. J. Bünemann, W. Weber and F. Gebhard, Phys. Rev. B57, 6896 (1998).

73. N.E. Zein, Phys. Rev. B52, 11813 (1995).

74. W. Metzner and D. Vollhardt, Phys. Rev. Lett. 59, 121 (1987).

75. W. Metzner and D. Vollhardt, Phys. Rev. B37, 7382 (1988)

76. G. Seibold, E. Sigmund, and V. Hizhnyakov, Phys. Rev. B57, 6937 (1998).

77. G. Kotliar and A.E. Ruckenstein, Phys. Rev. Lett. 57, 1362 (1986)

78. T. Li, P. Wölfle, and P.J. Hirschfeld, Phys. Rev. B40, 6817 (1989).

79. J.W. Rasul and T. Li, J. Phys. 21, 5119 (1988).

80. M. Lavagna, Phys. Rev. B41, 142 (1990).

81. E. Arrigoni and G.C. Strinati, Phys. Rev. Lett. 71, 3178 (1993)

82. E. Arrigoni and G.C. Strinati, Phys. Rev. B52, 2428 (1995).

83. F. Gebhard, Phys. Rev. B41, 9452 (1990).

84. T. Li, P. Wölfle, and P.J. Hirschfeld, Phys. Rev. B40, 6817 (1989); R. Frésard and P. Wölfle, Int. J. Mod. Phys. B6, 237 (1992).

85. M. Bak and R. Micnas, J. Phys.: Condens. Matter 10, 9029 (1998).

86. P. Ring and P. Schuck, The Nuclear Many-Body Problem, Springer-Verlag, New York (1980).

87. J. Blaizot and G. Ripka, Quantum Theory of Finite Syatems, MIT Press (1986).

88. H. Yokoyama and H. Shiba, J. Phys. Soc. Jpn. 56, 3582 (1987).

89. G. Fano, F. Ortolani, and A. Parola, Phys. Rev. B42, 6877 (1990).

90. G.D. Mahan, Many Particle Physics, Plenum, New York (1990).

91. G. Seibold and J. Lorenzana, Phys. Rev. B69, 134513 (2004).

92. A.K. McMahan, J.F. Annett, and R.M. Martin, Phys. Rev. B42, 6268 (1990).

93. M.S. Hybertsen, M. Schluter, and N.E. Christiansen, Phys. Rev. B39, 9028 (1989).

94. M. King, Z.X. Shen, D.S. Dessau, D.S. Marshall, C.H. Park, W.E. Spicer, J.L. Peng, Z.Y. Li, and R.L. Greene, Phys. Rev. Lett. 73, 298 (1994).

95. A.M. Ole's and J. Zaanen, Ann. Phys. (Leipzig) 5, 224 (1996)

96. J. Lorenzana and L. Yu, Phys. Rev. Lett. 70, 861 (1993).

97. S.A. Kivelson, E. Fradkin, and V.J. Emery, Nature 393, 550 (1998)

98. Y.J. Uemura, Solid State Commun. 120, 347 (2001).

99. P. Prelovsek, T. Tohyama, and S. Maekawa, Phys. Rev. B64, 052512 (2001).

100. N.P. Ong, Phys. Rev. B43, 193 (1991). 
101. T. Timusk, S.L. Herr, K. Ramaras, C.D. Porter, D.B. Tanner, D.A. Bonn, J.D. Garrett, C.V. Stager,J.E. Greedan, and M. Reedyk, Phys. Rev. B38, 6683 (1988).

102. S. Caprara, C.Di Castro, S. Fratini, and M. Grilli, Phys. Rev. Lett. 88, 147001 (2002).

103. M. Terauchi and M. Tanaka, Micron 30, 371 (1999).

104. J.M. Ginder, M.G. Roe, Y. Song, R.P. McCall, J.R. Gaines, E.Ehrenfreund, and A.J. Epstein, Phys. Rev. B37, 7506 (1988).

105. J.M. Leng, J.M. Ginder, W.E. Farneth, S.I. Shah, and A.J. Epstein, Phys. Rev. B43, 10582 (1991).

106. C.C. Homes, J.M. Tranquada, O. Li, A.R. Moodenbaugh, and D.J. Buttrey, Phys. Rev. B67, 184516 (2003).

107. F.C. Zhang and T.M. Rice, Phys. Rev. B37, 3759 (1988).

108. K. Yonemitsu, A.R. Bishop, and J. Lorenzana, Phys. Rev. Lett. 69, 965 (1992).

109. T. Tohyama, S. Nagai, Y. Shibata, and S. Maekawa, Phys. Rev. Lett. 82, 4910 (1999).

110. G.B. Martins, C. Gazza, J.C. Xavier, A. Feiguin, and E. Dagotto, Phys. Rev. Lett. 84, 5844 (2000).

111. V.I. Anisimov, M.A. Korotin, A.S. Mylnikova, A.V. Kozhevnikov, and J. Lorenzana, Phys. Rev. Lett. B70, 172501 (2004).

112. E. Pavarini, I. Dasgupta, T. Saha-Dasgupta, O. Jepsen, and O. K. Andersen, Phys. Rev. Lett. 87, 047003 (2001).

113. S.R. White and D.J. Scalapino, Phys. Rev. B60, R753 (1999).

114. Marcus Fleck, Eva Pavarini, and Ole Krogh Andersen, cond-mat/0102041.

115. K. Machida and M. Ichioka, J. Phys. Soc. Jpn. 68, 2168 (1999).

116. B. Valenzuela, M.A.H. Vozmediano, and F. Guinea, Phys. Rev. B62, 11312 (2000).

117. B. Normand and A.P. Kampf, Phys. Rev. B65, 020509 (2001).

118. R. Coldea, S.M. Hayden, G. Aeppli, T.G. Perring, C.D. Frost, T.E. Mason, S.W. Cheong, and Z. Fisk, Phys. Rev. Lett. 86, 5377 (2001).

119. M. Roger and J.M. Delrieu, Phys. Rev. B39, 2299 (1989)

120. H.J. Schmidt and Y. Kuramoto, Physica C167, 263 (1990).

121. F. Lema, J.M. Eroles, C.D. Batista, and E. Gagliano, Phys. Rev. B55, 15295 (1997).

122. J. Lorenzana, J. Eroles, and S. Sorella, Phys. Rev. Lett. 83, 5122 (1999).

123. M.A. Kastner, R.J. Birgeneau, G. Shirane, and Y. Endoh, Rev. Mod. Phys. 70, 897 (1998).
124. E. Manousakis, Rev. Mod. Phys. 63, 1 (1991).

125. H.A. Mook, Pengcheng Dai, S.M. Hayden, G. Aeppli, T.G. Perring, and F. Dogan, Nature 395, 580 (1998).

126. S.M. Hayden, H.A. Mook, Pengcheng Dai, T. G. Perring, and F. Dogan, Nature 429, 531 (2004).

127. J. Rossat-Mignod, L.P. Regnault, C. Vettier, P. Bourges, P. Burlet, J. Bossy, J.Y. Henry, and G. Lapertot, Physica C185-189, 86 (1991).

128. H.F. Fong, B. Keimer, P.W. Anderson, D. Reznik, F. Dogan, and A. Aksay, Phys. Rev. Lett. 75, 316 (1995).

129. P. Bourges, L.P. Regnault, Y. Sidis, and C. Vettier, Phys. Rev. B53, 876 (1996).

130. Pengcheng Dai, H.A. Mook, S.M. Hayden, G. Aeppli, T.G. Perring, R.D. Hunt, and F. Dogan, Science 284, 1344 (1999).

131. Pengcheng Dai, H.A. Mook, G. Aeppli, S.M. Hayden, and F. Dogan, Nature 406, 965 (2000).

132. D. Reznik, P. Bourges, L. Pintschovius, Y. Endoh, Y. Sidis, T. Matsui, and S. Tajima, Phys. Rev. Lett 93, 207003 (2004).

133. N.B. Christensen, D.F. McMorrow, H.M. Ronnow, B. Lake, S.M. Hayden, G. Aeppli, T.G. Perring, M. Mangkorntong, M. Nohara, and H. Takagi, Phys. Rev. Lett. 93, 207002 (2004).

134. J. Brinckmann and P.A. Lee, Phys. Rev. Lett. 82, 2915 (1999); Ying-Jer Kao, Qimiao Si, and K. Levin, Phys. Rev. B61, R11898 (2000); M.R. Norman, Phys. Rev. B61, 14751 (2000); F. Onufrieva and P. Pfeuty, Phys. Rev. B65, 054515 (2002).

135. S. Pailhes, Y. Sidis, P. Bourges, V. Hinkov, A. Ivanov, C. Ulrich, L.P. Regnault, and B. Keimer, Phys. Rev. Lett. 93, 167001 (2004).

136. C.D. Batista, G. Ortiz, and A.V. Balatsky, Phys. Rev. B64, 172508 (2001); F. Krüger and S. Scheidl, Phys. Rev. B67, 134512 (2003); E.W. Carlson, D.X. Yao, and D.K. Campbell, Phys. Rev. B70, 064505 (2004).

137. A.T. Boothroyd, D. Prabhakaran, P.G. Freeman, S.J.S. Lister, M. Enderle, A. Hiess, and J. Kulda, Phys. Rev. B67, 100407R (2003).

138. G. Seibold and J. Lorenzana, cond-mat/0509175.

139. J. Lorenzana, G. Seibold, and R. Coldea, Phys. Rev. B, in press, cond-mat/0507131.

140. J.M. Tranquada, private communication.

141. M. Vojta and T. Ulbricht, Phys. Rev. Lett; 93, 127002 (2004); G.S. Uhrig, K.P. Schmidt, and M. Grüninger, Phys. Rev. Lett. 93, 267003 (2004).

142. S. Sachdev, Rev. Mod. Phys. 75, 913 (2003).

143. A.T. Boothroyd, P.G. Freeman, D. Prabhakaran, A. Hiess, M. Enderle, J. Kulda, and F. Altorfer, Phys. Rev. Lett. 91, 257201 (2003). 